Article

\title{
Geochemical Contrasts between Late Triassic Rb-Rich and Barren Pegmatites from Ningshan Pegmatite District, South Qinling Orogen, China: Implications for Petrogenesis and Rare Metal Exploration
}

\author{
Xiao Nie ${ }^{1}$, Zongqi Wang ${ }^{1}$, Lei Chen ${ }^{1}{ }^{*}$, Gang Wang ${ }^{1}$ and Zengda $\mathrm{Li}^{2}$ \\ 1 Ministry of Natural Resources Key Laboratory of Metallogeny and Mineral Assessment, Institute of Mineral \\ Resources, Chinese Academy of Geological Sciences, Beijing 100037, China; niexiao369@gmail.com (X.N.); \\ kcwzq@protonmail.com (Z.W.); wanggang0315@126.com (G.W.) \\ 2 Department of Exploration Technology, Minmetals Exploration and Development Co., Ltd., Beijing 100010, \\ China; li_zda@163.com \\ * Correspondence: chenlei@cags.ac.cn
}

Received: 22 May 2020; Accepted: 26 June 2020; Published: 28 June 2020

check for updates

\begin{abstract}
The Ningshan pegmatite district in the South Qinling Orogen hosts numerous Rb-(Be) mineralized pegmatites. In this study, whole-rock geochemistry, mineral geochemistry, and zircon $\mathrm{U}-\mathrm{Pb}$ isotopes of the $\mathrm{Rb}$-rich and barren pegmatites were determined. The barren pegmatites consist mainly of muscovite, microcline, albite, quartz, and garnet, whereas the Rb-rich pegmatites are mainly composed of muscovite, albite, quartz, and beryl, with minor chrysoberyl, cassiterite and columbite-group mineral. The muscovite and albite are the main Rb-bearing minerals. The $\mathrm{U}-\mathrm{Pb}$ zircon dating of the $\mathrm{Rb}$-rich and barren pegmatites yielding an age of 212-203 Ma, which is similar to that of the neighboring two-mica monzogranites distributed in the Ningshan area. Compared with the two-mica monzogranites, geochemical features, such as the $\mathrm{Zr} / \mathrm{Hf}, \mathrm{Rb} / \mathrm{Sr}$ and $\mathrm{Nb} / \mathrm{Ta}$ ratios and trace element contents indicated that the $\mathrm{Rb}$-rich and barren pegmatites derived from fractionation of the two-mica monzogranites. In combination, the current and previous results suggest that the fractionation of the two-mica monzogranites caused the generation of the affinitive residual melts that, finally, crystallized to form the pegmatites. Compared to those from the barren pegmatites, the apatite from the $\mathrm{Rb}$-rich pegmatites have higher $\mathrm{MnO}$ (14.51-19.12 wt.\%) and $\mathrm{Cl}(0.12-0.16 \mathrm{wt} . \%)$ contents and lower $\mathrm{F} / \mathrm{Cl}$ rartios (20-29). We conclude that these differences reflect unique geochemical signatures, and the geochemical composition of the apatite can be used as exploration guidance for rare metal-rich pegmatites.
\end{abstract}

Keywords: whole-rock geochemistry; mineral geochemistry; zircon $\mathrm{U}-\mathrm{Pb}$ age; pegmatite; rubidium mineralization; South Qinling Orogen

\section{Introduction}

Rare metals, such as lithium, beryllium, cesium, rubidium, tin, niobium, tantalum, and uranium, are important to the economy and defense of the country. Today, many geologists are increasingly paying attention to granitic pegmatites since they are important sources of rare metals [1]. The classification of rare-element pegmatite into LCT (rich in $\mathrm{Li}, \mathrm{Cs}$, and $\mathrm{Ta}$, in addition to $\mathrm{Rb}, \mathrm{Be}, \mathrm{Sn}, \mathrm{B}, \mathrm{P}$, and F), NYF (rich in $\mathrm{Nb}, \mathrm{Y}$ and $\mathrm{F}$, in addition to $\mathrm{Be}, \mathrm{REE}, \mathrm{Sc}, \mathrm{Ti}, \mathrm{Zr}$, Th, and $\mathrm{U}$ ), and mixed petrogenetic families [2-4] is the most widely applied today. LCT family is suggested to be associated usually with I- or S-type granite derived from a sedimentary source in a late- or post-orogenic setting. The NYF family is suggested to be associated with A-type granite derived from a mixed source of lower crust 
and mantle in a post- or anorogenic setting [3,5]. As regards pegmatite genesis, two genetic models exist. The most accepted model assumes an igneous origin, with most pegmatites originating from volatile-rich residual melts derived from the crystallization of parental granitic magmas. In this model, pegmatite is assumed to be linked genetically with fertile granite(s) exposed at the surface or buried at depth [1,3,5-9]. The other model assumes an anatectic origin (e.g., in migmatitic terranes), whereby the pegmatites form by direct partial melting of metasedimentary rocks, and have no petrogenetic relation to the neighboring granite(s) [10-20].

Three large granitic pegmatite regions are distributed in the Qinling Orogenic Belt in China: the Shangnan, Baoji, and Ningshan pegmatite districts. Detailed studies have been conducted on the Shangnan pegmatite district, with the classification, internal structure, mineral assemblage, mineralization characteristics, metallogenetic epoch, and genetic relationships between granites and pegmatites having been reported [21-25]. In contrast, the Ningshan pegmatite district, located in South Qinling Orogen, is poorly investigated. All the pegmatites in the Ningshan district are distributed in the outer contact zone on the south side of the Triassic granitoids in the Wulong-Ningshan area, but the pegmatite-granite genetic relationship remains puzzling. Notably, $\mathrm{Rb}$ and Be enrichment is observed in some of the pegmatites. It is therefore very important to understand the distinct geochemical patterns between the barren and $\mathrm{Rb}$-rich pegmatites since it can not only enhance the knowledge about the pegmatite petrogenesis but also provides useful information for rare metal exploration regionally.

In this study, we performed mineralogical, geochemical, and zircon $\mathrm{U}-\mathrm{Pb}$ isotopic data on Lizhaping, Dazengxigou, Xinjian barren pegmatites, and Xinjian Rb-rich pegmatites from the Ningshan pegmatites district. Subsequently, by undertaking a detailed geochemical comparison among the barren pegmatites, Rb-rich pegmatites, and the neighboring granites, we attempted to clarify the petrogenesis of the pegmatites. Furthermore, by comparing apatite composition of the barren pegmatites with that of $\mathrm{Rb}$-rich pegmatites, we attempted to evaluate the application of apatite as an indicator mineral in rare metal exploration.

\section{Geological Setting}

The Qinling Orogenic Belt (QOB) is located in central China and is bounded to the north by the Lingbao-Lushan Fault and to the south by the Mianlue-Bashan Fault, connecting the North (NCB) and South China Block (SCB) (Figure 1a) [26-28]. As proposed by Meng and Zhang (1999) [26], two important sutures are located in the QOB, namely, the Shangdan suture in the north and the Mianlue suture in the south (Figure 1b). It is generally accepted that the Shangdan suture records the middle Paleozoic northward subduction of the Shangdan (Paleo-Qinling) Ocean along the southern margin of the Norh Qinling Belt (NQB) and the amalgamation of the NQB and South Qinling Belt (SQB) $[27,29]$. The Mianlue suture represents the late Paleozoic Mianlue (Paleo-Tethys) Ocean that closed in the early Mesozoic, followed by the final collision between the SCB and the united NCB-SQB [30-33]. The main geologic framework of the QOB was built up by the middle Paleozoic and early Mesozoic subduction-collision events of the NCB (including the NQT), SQB, and SCB at the Shangdan and Mianlue sutures [26,27,32].

Tectonically, with the Shangdan and Mianlue sutures and the Luonan-Luanchuan fault (LL), the QOB is divided into, from north to south, the southern margin of the NCB, NQB, SQB, and the northern margin of the SCB (Figure 1b) [30,34]. The SQB is characterized by south-verging thrusts and folds, showing an imbricated thrust-fold system [35]. The SQB consists of Proterozoic metamorphic sequences, late Paleozoic sedimentary sequences [35], and numerous Triassic granitoids [36-39] (Figure 1b). These Triassic granitoids intruded into the SQB as a result of the subduction and collision between the SQB and SCB along the Mianlue suture (M) [36-39]. From west to east, the granitoids have been classified into three suites, which are the Guangtoushan, Huayang-Wulong-Ningshan (including the Huayang, Wulong, Laocheng, Xichahe, and Yanzhiba plutons), and Dongjiakou suites (Figure 1b). These granitoids extends along the SQB roughly parallel to the Mianlue suture, forming a ca. $400 \mathrm{~km}$ long granitoid belt. 


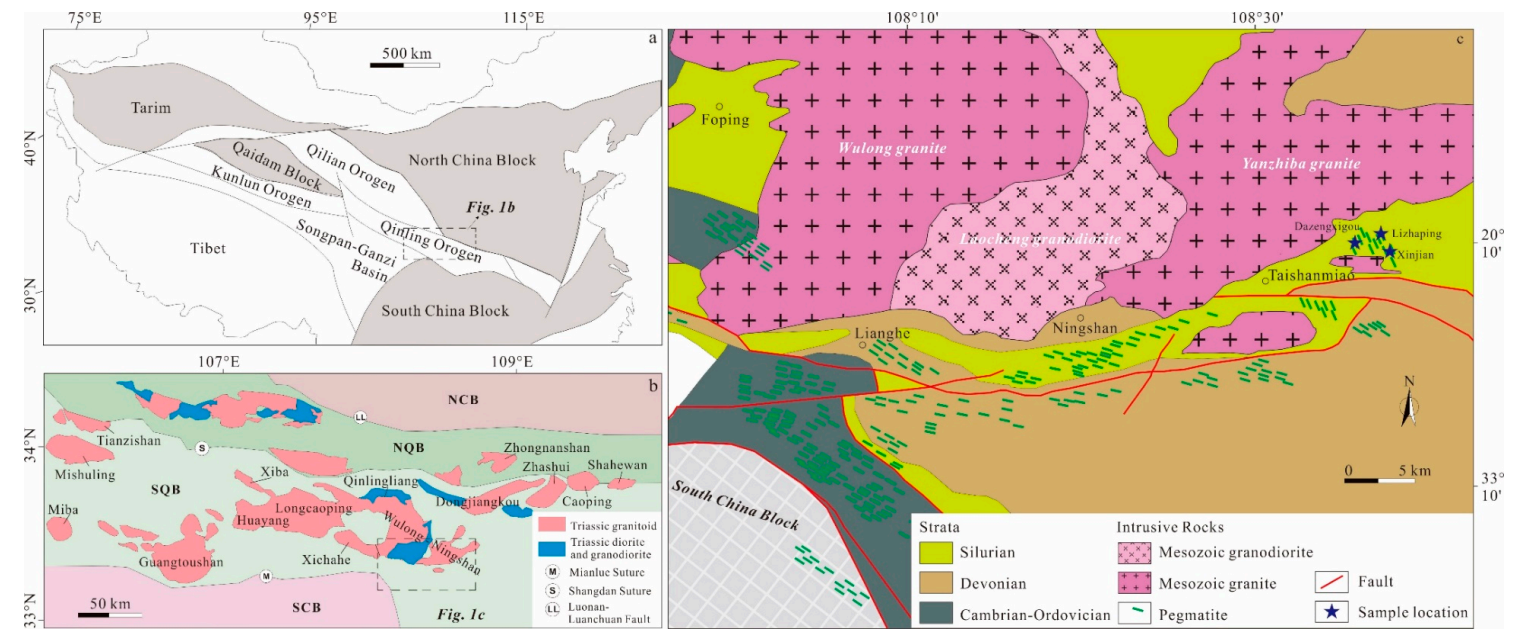

Figure 1. (a) simplified tectonic map of China showing major tectonic units and the location of the Qinling Orogen; (b) geological map showing the distribution of the granitoids in the middle segment of the Qinling Orogen (modified from [36,37,39,40]; (c) geological sketch map of Ningshan pegmatite district (modified from [21]). Abbreviations: NCB = North China Block; NQB = North Qinling Belt; SQB = South Qinling Belt; SCB = South China Block.

Three large granitic pegmatite regions are distributed in the Qinling Orogenic Belt, namely, the Shangnan, Baoji, and Ningshan pegmatite districts. The Shangnan and Baoji pegmatite districts are distributed north of the Shangdan suture. The Ningshan pegmatite district is located on the south of the Triassic granitoids in the Wulong-Ningshan area in the middle segment of SQB (Figure 1b,c) [21,22]. In terms of rare metal mineralization, mainly $\mathrm{Nb}, \mathrm{Ta}, \mathrm{Be}, \mathrm{Rb}$, and $\mathrm{Li}$ mineralization developed in the Shangnan pegmatite district; mainly $\mathrm{Nb}, \mathrm{U}, \mathrm{REE}$ mineralization developed in the Baoji pegmatite district; and mainly $\mathrm{Be}$, and $\mathrm{Rb}$ mineralization are developed in the Ningshan pegmatite district $[21,22]$.

\section{Pegmatite and Sample Description}

The pegmatites we studied are located $5 \mathrm{~km} \mathrm{NE}$ of the township of Taishanmiao, and are distributed to the south of the Yanzhiba pluton (Figure 1c). Previous geological surveys indicated that these pegmatites have high $\mathrm{Rb}$ content, with muscovite mainly being the Rb-bearing mineral. Be mineralization is also found in some Rb-rich pegmatites [41].

In this study, we collected more than 20 representative samples from barren pegmatites along the road-cuts of Lizhaping (location: $33^{\circ} 20^{\prime} 10^{\prime \prime}, 108^{\circ} 36^{\prime} 17^{\prime \prime}$ ), Dazengxigou (location: 33 $3^{\circ} 19^{\prime} 16^{\prime \prime}, 108^{\circ} 34^{\prime} 2^{\prime \prime}$ ) and Xinjian (location: $33^{\circ} 17^{\prime} 13^{\prime \prime}, 108^{\circ} 35^{\prime} 27^{\prime \prime}$ ) country. These pegmatites are ca. 20-30 $\mathrm{m}$ long and $20 \mathrm{~cm}-2 \mathrm{~m}$ wide (Figure $2 \mathrm{a}, \mathrm{b}$ ). Some of the barren pegmatites intruded into the two-mica monzogranites, with a progressive evolution contact (Figure 2a). These pegmatites have nonsignificant internal texture, and are composed of biotite, muscovite, K-feldspar, albite, and quartz. Other barren pegmatites are hosted in the sericite slate and quartz sandstone-siltstone of lower-middle Silurian Meiziya Formation (Figure 2b). These pegmatites have irregular internal texture, and two discontinuous mineralogical-textural zones can be distinguished. The outermost zone is mainly composed of medium-fine grained muscovite, K-feldspar, albite, and quartz (Figure 2c,d). The granophyric albite-quartz intergrowth margins are partially visible in the outermost zone. The inward zone mainly contains medium-coarse grained muscovite, microcline, albite, quartz, and garnet (Figure 2e,f). 

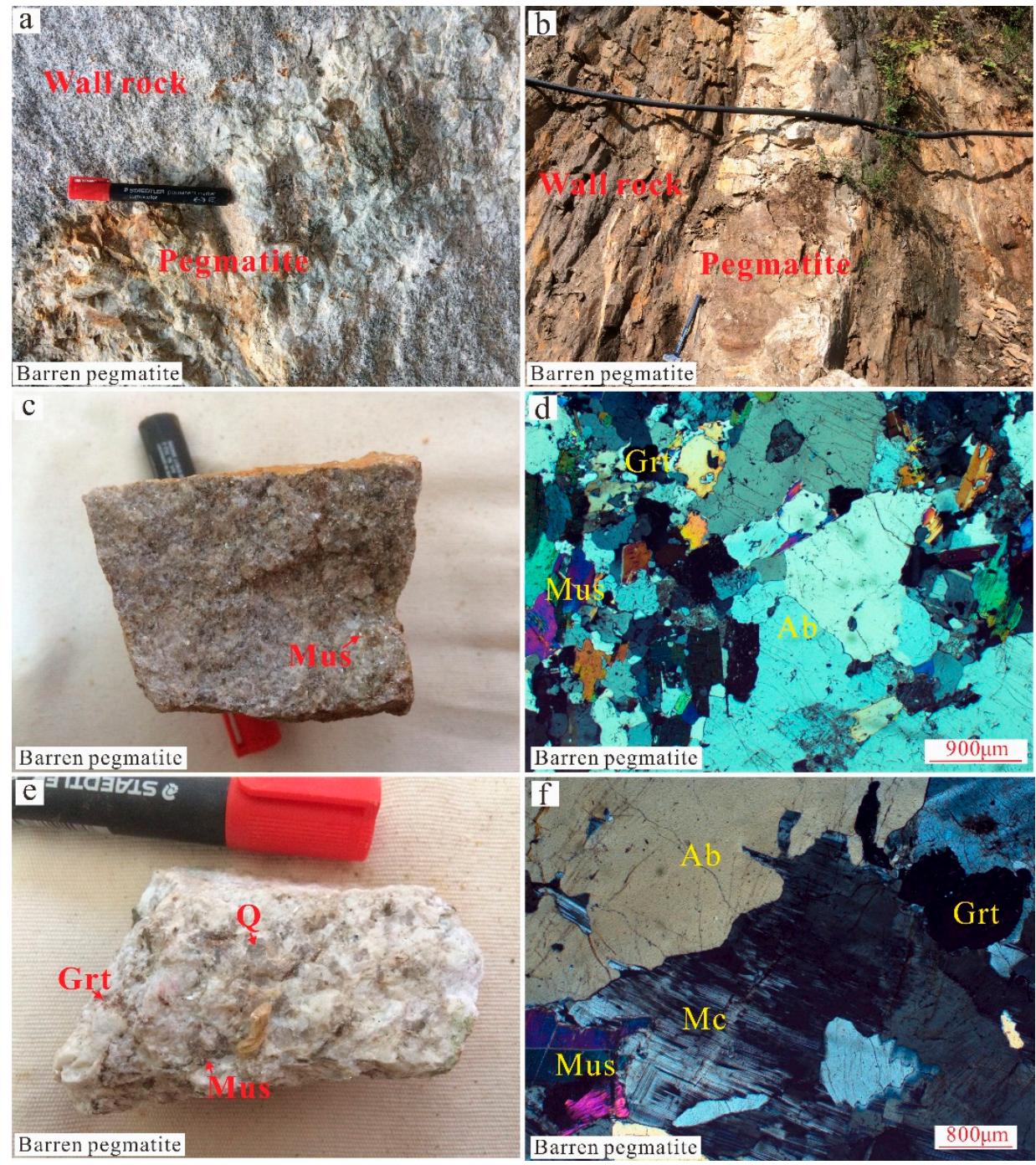

Figure 2. Field-, macro- and micro-photographs of the barren pegmatite dikes. (a,b) outcrops of the barren pegmatite; (c,d) photographs and micrographs of the barren pegmatite in outermost zone, with medium-fine-grained texture; (e,f) photographs and micrographs of the barren pegmatite in inward zone, with medium-coarse-grained texture. Abbreviation: Mus = muscovite; $Q=$ quartz; Grt = garnet; $\mathrm{Mc}=$ microcline; $\mathrm{Ab}=$ albite .

More than 10 representative samples from $\mathrm{Rb}$-rich pegmatites along the road-cuts of the Xinjian country were collected. These pegmatites are ca. 20-30 m long and 1-3 m wide (Figure 3a). Wall rocks of the Rb-rich pegmatites are mainly the lower-middle Silurian Meiziya Formation (Figure 3a). The internal texture of the Rb-rich pegmatites is nonsignificant. The Xinjian Rb-rich pegmatites are mainly coarse-medium grained (Figure $3 b$ ), and mainly composed of muscovite, albite, quartz, and beryl (Figure $3 c, d$ ). The beryl forms euhedral to subhedral crystals varying in size from several hundred micrometers to several millimeters (Figure 3d). Other minerals include chrysoberyl, cassiterite, and columbite-group mineral, which commonly occur as accessory minerals (Figure $3 \mathrm{~d}-\mathrm{f}$ ). 


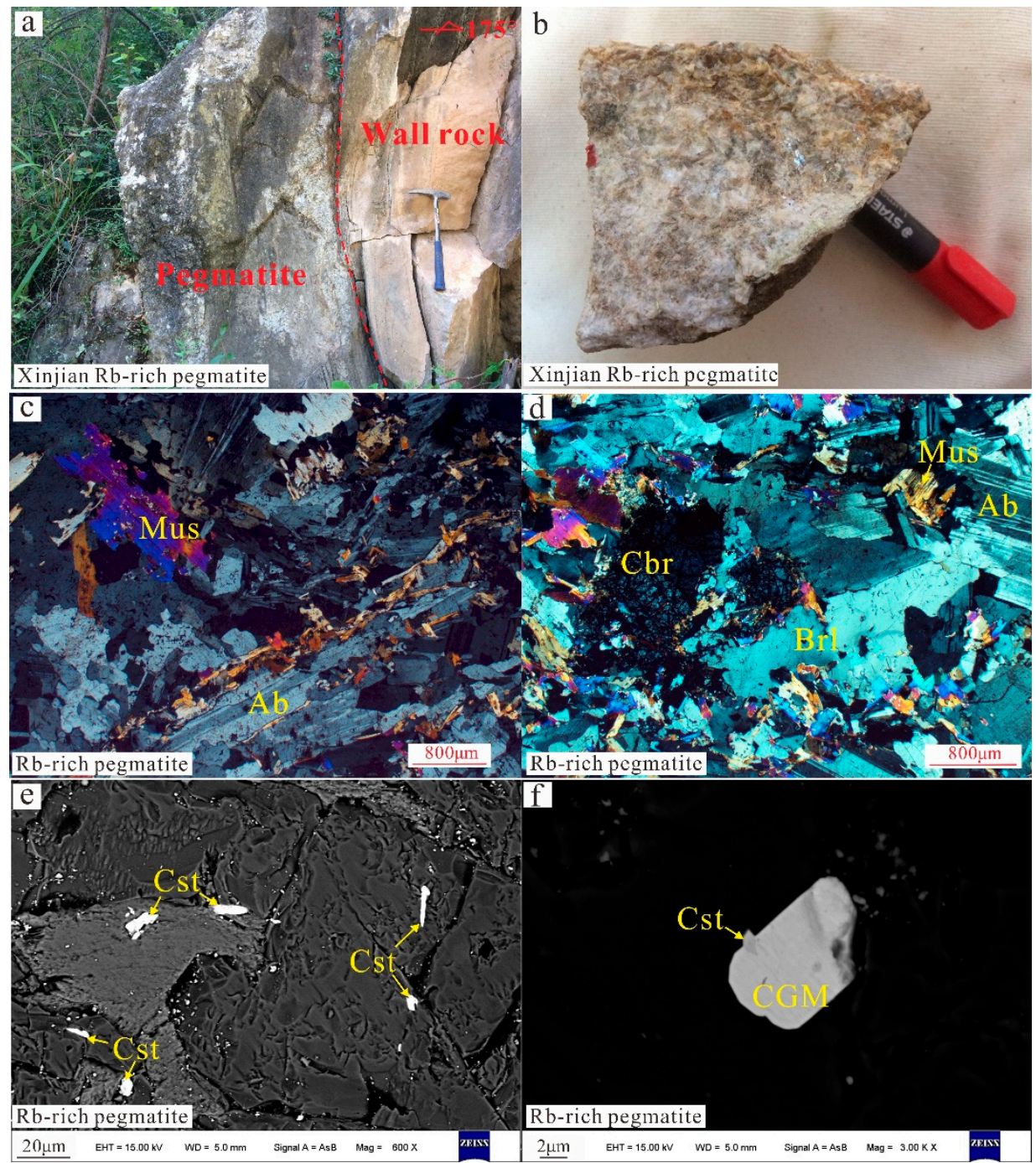

Figure 3. Field-, macro- and micro-photographs of the Rb-rich pegmatite dikes. (a) outcrops of the Xinjian $\mathrm{Rb}$-rich pegmatite, and showing sharp contact relationships between the pegmatites and their wall rocks; (b) photographs of the Xinjian Rb-rich pegmatite in hand specimen, with coarse -medium grained texture; (c,d) micrographs of the Xinjian Rb-rich pegmatites; $(\mathbf{e}, \mathbf{f})$ BSE images of the Xinjian $\mathrm{Rb}$-rich pegmatites. Abbreviation: Mus = muscovite; $\mathrm{Ab}=$ albite; $\mathrm{Brl}=$ beryl; $\mathrm{Cbr}=$ chrysoberyl; Cst $=$ cassiterite $; \mathrm{CGM}=$ columbite-group mineral.

\section{Analytical Methods}

\subsection{Whole-Rock Compositional Analysis}

Fresh whole-rock samples from Rb-rich muscovite-albite (Mus-Ab) pegmatite and inward zone of barren muscovite-microcline-albite (Mus-Mc- $\mathrm{Ab}$ ) pegmatite were trimmed to remove weathered surfaces, cleaned with deionized water, crushed in a steel jawcrusher and then powdered to $<200$ mesh using an agate mill. Sample preparation and whole-rock major and trace elements were determined at the National Research Center for Geoanalysis, Chinese Academy of Geological Sciences (CAGS), Beijing, China. Major element concentrations were analyzed by X-ray fluorescence (XRF) spectrometry, with analytical uncertainties of $\leq 0.5 \mathrm{wt} . \%$. Ferric and ferrous iron measurements were performed via wet chemical analysis (titration). The analytical precision for major oxides was based on certified standards (GSR-1, GSR-3). Trace elements were determined using ICP-MS. The analytical uncertainties 
are $10 \%$ when the element abundance is $<10 \mathrm{ppm}$, and around $5 \%$ when the element abundance $>10 \mathrm{ppm}$.

\subsection{Mineral Compositions Analysis}

Back-scattered electron (BSE) images and quantitative chemical analyses of minerals were performed from polished thin sections using a JEOL JXA-8100 electron probe micro-analyzer (EPMA, JEOL Ltd., Akishima, Tokyo, Japan). Silicate minerals and apatite from barren Mus-Mc-Ab pegmatite were obtained at Beijing Research Institute of Uranium Geology, China. Silicate mineral and apatite from Rb-rich Mus-Ab pegmatite at Institute of Geology and Geophysics, Chinese Academy of Sciences, Beijing. The operating conditions were set at an acceleration voltage of $15 \mathrm{kV}$, a probe current of $10 \mathrm{nA}$ and a beam diameter of $5 \mu \mathrm{m}$. Element peaks and off-peak backgrounds were measured with counting times of $10 \mathrm{~s}$ and $5 \mathrm{~s}$, respectively, except for $\mathrm{F}, \mathrm{Cl}(20$ and $10 \mathrm{~s})$ and $\mathrm{Rb}$ (20 and $10 \mathrm{~s})$. Standards used for analyses were as follows: diopside $(\mathrm{Mg}, \mathrm{Si}, \mathrm{Ca})$, rutile $(\mathrm{Ti})$, plagioclase $(\mathrm{Al})$, garnet $(\mathrm{Fe})$, rhodonite $(\mathrm{Mn})$, albite $(\mathrm{Na})$, sanidine $(\mathrm{K})$, apatite $(\mathrm{P})$, pollucite $(\mathrm{Rb})$, durango $(\mathrm{F})$, tugtupite $(\mathrm{Cl})$. Data reduction was performed using the internal ZAF matrix correction of JEOL.

\subsection{Zircon CL Images and LA-ICP-MS U-Pb Dating}

Zircons were separated from the three least altered samples (LZP01 and DZXG03: barren Mus-Mc-Ab pegmatite, XJ01-4: Rb-rich Mus-Ab pegmatite) by standard density and magnetic separation techniques followed by hand-picking under a binocular microscope for purification. Prior to the in-situ zircon U-Pb isotope analyses, the morphology and internal structure of these zircons were studied using a binocular microscope and cathodoluminescence (CL) images, which were used as guides during the selection of the U-Pb dating spots. The zircon $\mathrm{U}-\mathrm{Pb}$ dating and trace element analyses were conducted synchronously employing laser ablation inductively coupled plasma mass spectrometry (LA-ICP-MS) at the Institute of Mineral Resources, CAGS, in Beijing, China. Detailed information on the operating conditions for the laser ablation system, the ICP-MS instrument, and the data reduction process have been described previously by Liu et al. $(2008,2010)$ and Hou et al. (2009) [42-44].

\section{Results}

\subsection{Whole-Rock Geochemistry}

We analyzed six pegmatite samples from barren Mus-Mc-Ab pegmatite (LZP03-1, LZP03-2, DZXG01-1, DXZG01-2, DZXG01-3, and XJ01-3) and two sample from Rb-rich Mus-Ab pegmatite (XJ01-1 and XJ01-2) for major and trace element compositions (Table 1).

The barren Mus-Mc-Ab and Rb-rich Mus-Ab pegmatite had similar major element compositions. They are characterized by high $\mathrm{SiO}_{2}$ (72.27-76.71 wt.\%), $\mathrm{Al}_{2} \mathrm{O} 3$ (13.46-17.57 wt.\%), and alkali $\left(\mathrm{K}_{2} \mathrm{O}+\mathrm{Na}_{2} \mathrm{O}=7.12-10.08\right.$ wt. $\left.\%\right)$ contents. In the A/NK vs. A/CNK diagram, they plot in the peraluminous field (Figure 4). Nevertheless, the trace element compositions of barren Mus-Mc-Ab and Rb-rich Mus-Ab pegmatite are significantly different. The total REE ( $\left.\sum R E E\right)$ concentrations of barren Mus-Mc-Ab pegmatite ranged from 17.84 to $51.96 \mathrm{ppm}$, and the chondrite-normalized REE diagrams are characterized by seagull-like normalized REE pattern, with significant negative Eu anomalies $\left(\mathrm{Eu} / \mathrm{Eu}^{*}=0.12-0.37\right)$ and low $(\mathrm{La} / \mathrm{Yb})_{\mathrm{N}}$ ratios $(1.58-3.14$; Figure 5a). On the primitive mantle-normalized spider diagrams (Figure $5 b$ ), most barren Mus-Mc-Ab pegmatites show positive in $\mathrm{Rb}$ and $\mathrm{U}$ anomalies, and negative anomalies of $\mathrm{Ba}, \mathrm{Nb}, \mathrm{Sr}, \mathrm{Zr}$, and Ti. In contrast, $\mathrm{Rb}$-rich Mus- $\mathrm{Ab}$ pegmatite have much lower $\sum$ REE concentrations (4.74-4.94 ppm) and further display a light REE $(\mathrm{LREE})$ enrichment $\left[(\mathrm{La} / \mathrm{Yb})_{\mathrm{N}}=(27.67-35.50)\right]$ without significant Eu anomalies (Figure 5a). Their trace element abundance diagrams are characterized by pronounced enrichment in $\mathrm{Rb}, \mathrm{Nb}, \mathrm{P}, \mathrm{Sr}$, and $\mathrm{Hf}$ (Figure 5b). 
Table 1. Major (wt.\%) and trace elements (ppm) of the barren Mus-Mc-Ab and Rb-rich Mus-Ab pegmatite.

\begin{tabular}{|c|c|c|c|c|c|c|c|c|}
\hline \multirow{2}{*}{ Sample } & \multicolumn{6}{|c|}{ Barren Pegmatite } & \multicolumn{2}{|c|}{ Rb-Rich Pegmatite } \\
\hline & LZP03-1 & LZP03-2 & DZXG01-1 & DZXG01-2 & DZXG01-3 & XJ01-3 & XJ01-1 & $\mathrm{XJ} 01-2$ \\
\hline $\mathrm{SiO}_{2}$ & 75.05 & 72.27 & 76.71 & 74.58 & 74.73 & 73.87 & 72.46 & - \\
\hline $\mathrm{Al}_{2} \mathrm{O}_{3}$ & 14.50 & 16.34 & 13.46 & 14.89 & 15.03 & 14.75 & 17.57 & - \\
\hline $\mathrm{MgO}$ & 0.10 & 0.16 & 0.10 & 0.11 & 0.13 & 0.11 & 0.04 & - \\
\hline $\mathrm{Na}_{2} \mathrm{O}$ & 2.61 & 2.96 & 4.03 & 5.21 & 5.31 & 3.93 & 4.18 & - \\
\hline $\mathrm{K}_{2} \mathrm{O}$ & 5.76 & 5.84 & 4.17 & 3.00 & 2.12 & 6.15 & 2.94 & - \\
\hline $\mathrm{P}_{2} \mathrm{O}_{5}$ & 0.08 & 0.10 & 0.12 & 0.10 & 0.15 & 0.06 & 0.29 & - \\
\hline $\mathrm{TiO}_{2}$ & 0.07 & 0.11 & 0.06 & 0.05 & 0.07 & 0.09 & 0.04 & - \\
\hline $\mathrm{CaO}$ & 0.73 & 0.80 & 0.76 & 0.95 & 1.17 & 0.68 & 0.47 & - \\
\hline $\mathrm{FeO}^{\mathrm{T}}$ & 0.50 & 0.67 & 0.42 & 0.62 & 0.52 & 0.39 & 0.21 & - \\
\hline $\mathrm{MnO}$ & 0.10 & 0.12 & 0.06 & 0.13 & 0.04 & 0.02 & 0.08 & - \\
\hline LOI & 0.67 & 0.96 & 0.55 & 0.54 & 0.77 & 0.46 & 1.61 & - \\
\hline Sum & 100.19 & 100.32 & 100.44 & 100.17 & 100.03 & 100.51 & 99.89 & - \\
\hline $\mathrm{A} / \mathrm{CNK}$ & 1.22 & 1.29 & 1.07 & 1.10 & 1.14 & 1.03 & 1.61 & - \\
\hline $\mathrm{A} / \mathrm{NK}$ & 1.38 & 1.46 & 1.21 & 1.26 & 1.36 & 1.12 & 1.74 & - \\
\hline $\mathrm{Na}_{2} \mathrm{O}+\mathrm{K}_{2} \mathrm{O}$ & 8.37 & 8.80 & 8.19 & 8.21 & 7.43 & 10.08 & 7.12 & - \\
\hline $\mathrm{Li}$ & 18.18 & 28.26 & 73.58 & 59.30 & 96.59 & 30.67 & 17.60 & 16.91 \\
\hline $\mathrm{Be}$ & 4.41 & 7.41 & 5.61 & 5.92 & 6.01 & 3.41 & 184.09 & 171.15 \\
\hline Sc & 3.54 & 8.04 & 2.56 & 3.24 & 3.52 & 6.35 & 0.88 & 0.75 \\
\hline $\mathrm{Ti}$ & 205.22 & 442.16 & 108.27 & 71.36 & 147.59 & 322.60 & 6.39 & 6.52 \\
\hline $\mathrm{V}$ & 4.16 & 2.73 & 0.69 & 0.62 & 3.11 & 3.16 & 1.04 & 0.99 \\
\hline $\mathrm{Cr}$ & 2.02 & 3.73 & 1.70 & 5.56 & 4.54 & 4.23 & 2.59 & 4.22 \\
\hline $\mathrm{Mn}$ & 707.55 & 875.58 & 453.39 & 908.04 & 259.98 & 145.26 & 501.43 & 505.98 \\
\hline Co & 0.19 & 0.25 & 0.20 & 0.10 & 0.10 & 0.28 & 0.05 & 0.06 \\
\hline $\mathrm{Ni}$ & 0.68 & 1.29 & 0.30 & 2.14 & 1.67 & 1.68 & 1.09 & 1.96 \\
\hline $\mathrm{Cu}$ & 0.03 & 9.20 & 2.31 & 0.64 & 0.03 & 3.40 & bdl. & bdl. \\
\hline $\mathrm{Zn}$ & 12.37 & 24.85 & 27.24 & 30.65 & 30.11 & 15.71 & 33.33 & 33.09 \\
\hline $\mathrm{Ga}$ & 20.23 & 31.20 & 14.37 & 15.55 & 15.25 & 22.62 & 22.46 & 21.48 \\
\hline $\mathrm{Rb}$ & 192.11 & 213.40 & 205.16 & 161.39 & 119.79 & 237.85 & 1043.29 & 1049.65 \\
\hline Cs & 6.25 & 7.61 & 11.26 & 17.85 & 12.77 & 10.35 & 657.84 & 650.40 \\
\hline $\mathrm{Sr}$ & 56.76 & 64.64 & 21.46 & 20.11 & 29.59 & 69.69 & 49.53 & 49.57 \\
\hline $\mathrm{Y}$ & 25.28 & 21.59 & 10.90 & 10.24 & 10.55 & 10.07 & 1.09 & 1.05 \\
\hline $\mathrm{Nb}$ & 16.24 & 29.67 & 12.82 & 14.80 & 15.92 & 17.61 & 133.74 & 125.07 \\
\hline $\mathrm{Ta}$ & 1.35 & 2.30 & 1.45 & 1.82 & 2.28 & 1.77 & 65.50 & 69.14 \\
\hline Mo & 0.09 & 0.10 & 0.13 & 0.07 & 0.03 & 0.13 & 0.09 & 0.11 \\
\hline $\mathrm{Sn}$ & 4.74 & 9.20 & 5.64 & 6.10 & 6.51 & 6.82 & 126.40 & 127.38 \\
\hline $\mathrm{Ba}$ & 229.45 & 232.63 & 28.52 & 8.16 & 9.20 & 151.98 & 9.95 & 9.35 \\
\hline $\mathrm{La}$ & 7.99 & 5.46 & 3.27 & 3.79 & 3.68 & 3.14 & 1.79 & 1.72 \\
\hline $\mathrm{Ce}$ & 17.66 & 11.85 & 6.73 & 7.81 & 7.94 & 5.45 & 1.57 & 1.52 \\
\hline $\operatorname{Pr}$ & 2.10 & 1.39 & 0.84 & 0.85 & 0.88 & 0.64 & 0.23 & 0.21 \\
\hline $\mathrm{Nd}$ & 7.62 & 5.15 & 3.11 & 2.82 & 3.22 & 2.33 & 0.81 & 0.77 \\
\hline $\mathrm{Sm}$ & 2.73 & 1.92 & 1.05 & 1.00 & 1.15 & 0.77 & 0.13 & 0.13 \\
\hline $\mathrm{Eu}$ & 0.26 & 0.23 & 0.09 & 0.04 & 0.06 & 0.21 & 0.04 & 0.04 \\
\hline $\mathrm{Gd}$ & 2.91 & 2.17 & 1.06 & 1.01 & 1.28 & 0.93 & 0.13 & 0.12 \\
\hline $\mathrm{Tb}$ & 0.64 & 0.51 & 0.25 & 0.25 & 0.29 & 0.21 & 0.01 & 0.01 \\
\hline Dy & 4.12 & 3.44 & 1.54 & 1.66 & 1.84 & 1.53 & 0.12 & 0.12 \\
\hline Ho & 0.76 & 0.66 & 0.32 & 0.29 & 0.32 & 0.30 & 0.01 & 0.01 \\
\hline $\mathrm{Er}$ & 2.18 & 1.98 & 1.01 & 0.81 & 0.86 & 0.94 & 0.05 & 0.05 \\
\hline $\mathrm{Tm}$ & 0.33 & 0.32 & 0.20 & 0.13 & 0.12 & 0.15 & bdl. & bdl. \\
\hline $\mathrm{Yb}$ & 2.34 & 2.37 & 1.48 & 0.96 & 0.84 & 1.10 & 0.04 & 0.04 \\
\hline $\mathrm{Lu}$ & 0.32 & 0.33 & 0.25 & 0.12 & 0.11 & 0.16 & bdl. & bdl. \\
\hline $\mathrm{Zr}$ & 43.47 & 34.19 & 20.55 & 28.61 & 31.46 & 15.50 & 26.73 & 27.33 \\
\hline Hf & 2.40 & 1.94 & 1.13 & 1.64 & 1.54 & 1.00 & 3.10 & 3.51 \\
\hline $\mathrm{W}$ & 1.39 & 2.51 & 1.12 & 1.76 & 1.81 & 1.65 & 4.27 & 4.32 \\
\hline $\mathrm{Tl}$ & 0.89 & 0.94 & 0.88 & 0.70 & 0.47 & 1.15 & 6.31 & 6.33 \\
\hline $\mathrm{Pb}$ & 36.48 & 35.78 & 33.69 & 24.30 & 20.63 & 39.61 & 22.60 & 21.98 \\
\hline Th & 5.67 & 3.75 & 1.79 & 2.18 & 2.34 & 2.02 & 1.01 & 0.96 \\
\hline $\mathrm{U}$ & 4.81 & 5.06 & 2.05 & 3.50 & 2.32 & 5.60 & 1.68 & 1.62 \\
\hline$\Sigma$ REE & 51.96 & 37.78 & 21.22 & 21.54 & 22.59 & 17.84 & 4.94 & 4.74 \\
\hline$(\mathrm{La} / \mathrm{Yb}) \mathrm{N}$ & 2.45 & 1.65 & 1.58 & 2.83 & 3.14 & 2.05 & 35.50 & 27.67 \\
\hline$\delta \mathrm{Eu}$ & 0.28 & 0.35 & 0.26 & 0.12 & 0.16 & 0.37 & 0.99 & 0.97 \\
\hline $\mathrm{Nb} / \mathrm{Ta}$ & 12.02 & 12.91 & 8.86 & 8.15 & 6.97 & 9.95 & 21.07 & 21.17 \\
\hline $\mathrm{Zr} / \mathrm{Hf}$ & 18.09 & 17.61 & 18.18 & 17.48 & 20.41 & 15.55 & 8.64 & 7.78 \\
\hline $\mathrm{Rb} / \mathrm{Sr}$ & 3.38 & 3.30 & 9.56 & 8.03 & 4.05 & 3.41 & 2.04 & 1.81 \\
\hline
\end{tabular}

Notes: $\mathrm{LOI}=$ loss on ignition; $\mathrm{A} / \mathrm{CNK}=$ molecular $\mathrm{Al}_{2} \mathrm{O}_{3} /\left(\mathrm{CaO}+\mathrm{Na}_{2} \mathrm{O}+\mathrm{K}_{2} \mathrm{O}\right) ; \mathrm{A} / \mathrm{NK}=$ molecular $\mathrm{Al}_{2} \mathrm{O}_{3} /\left(\mathrm{Na}_{2} \mathrm{O}+\right.$ $\mathrm{K}_{2} \mathrm{O}$ ); bdl. = below the detection limit. 


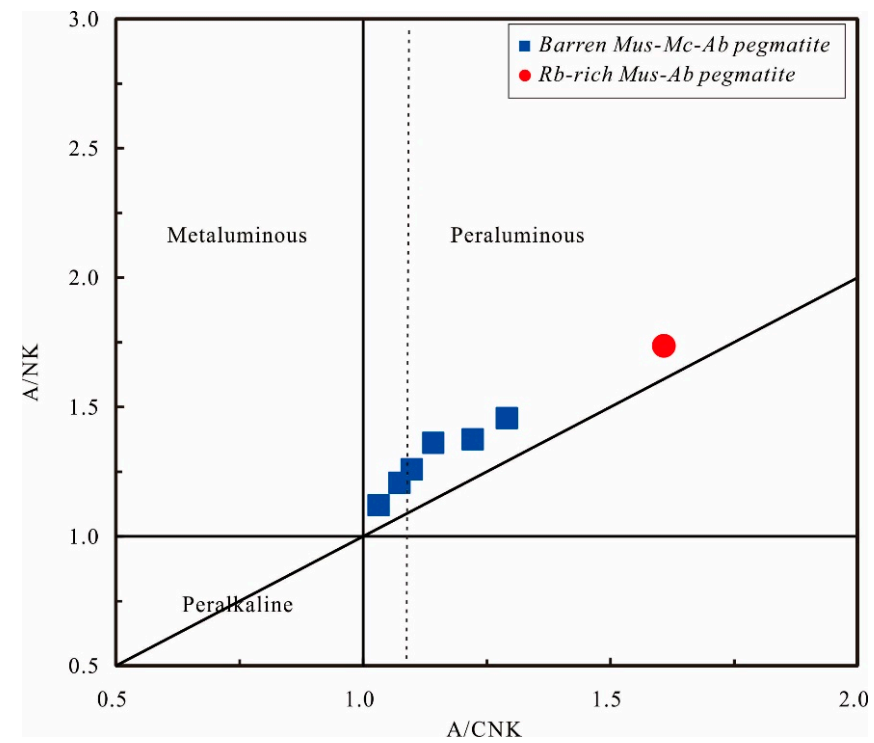

Figure 4. A/NK versus A/CNK plot (after Maniar and Piccoli, 1989) [45] indicating that the pegmatites are peraluminous.
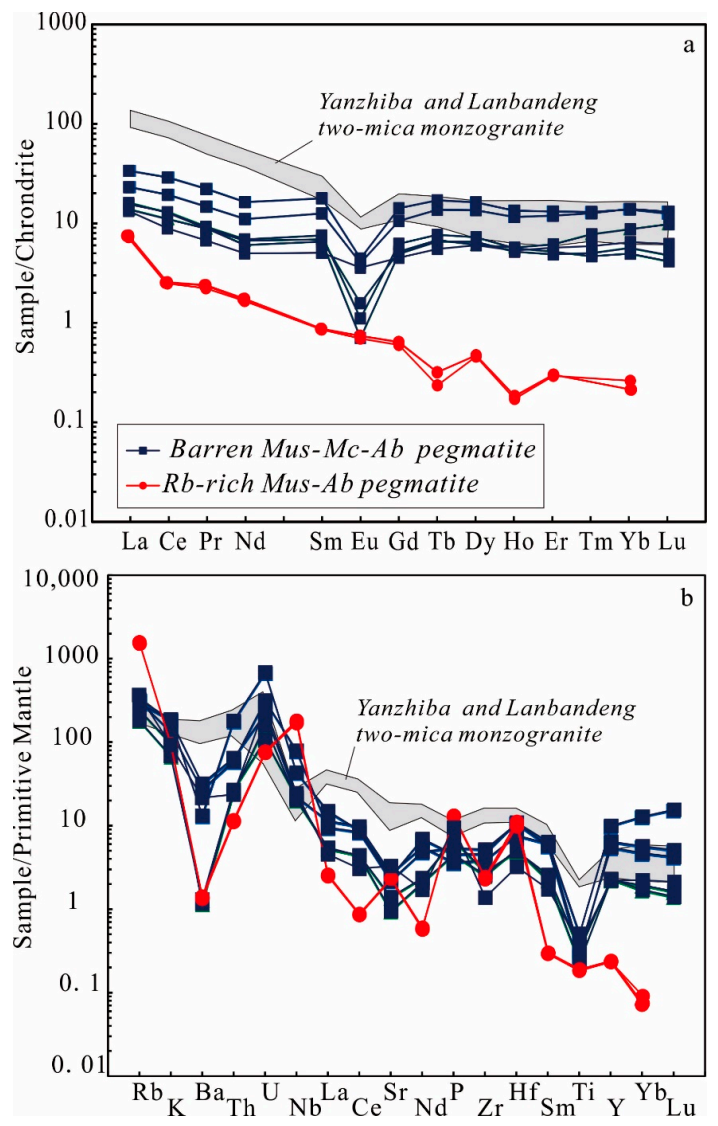

Figure 5. (a) chondrite normalized REE plot for pegmatites; (b) primitive mantle-normalized spider diagram for pegmatites. Data for normalization are cited from McDonough and Sun, 1989 [46]. Literature data of Yanzhiba and Lanbandeng two-mica monzogranites are from Jiang et al., 2010; Yang et al., $2012[47,48]$. 


\subsection{Mineral Geochemistry}

Analytical data of mica composition are summarized in Table 2. Micas in the studied samples are all compositionally homogeneous (Figure $6 \mathrm{a}, \mathrm{b}$ ). The micas from barren Mus-Mc-Ab pegmatite have high $\mathrm{K}_{2} \mathrm{O}$ (9.91-10.91 wt.\%) content, as well as low $\mathrm{F}(0-0.44 \mathrm{wt} . \%)$ and $\mathrm{Cl}(0-0.05 \mathrm{wt} . \%)$ contents. $\mathrm{The}^{\mathrm{Rb}} \mathrm{b}_{2} \mathrm{O}$ content was only detected ( $800 \mathrm{ppm}$ ) in one mica from Mus-Mc-Ab barren pegmatite. $\mathrm{K} / \mathrm{Rb}$ values of the barren pegmatites are more than 117. In contrast, the micas from Rb-rich Mus- $\mathrm{Ab}$ pegmatite have lower $\mathrm{K}_{2} \mathrm{O}$ (8.01-8.31 wt.\%), F (0.01-0.13 wt.\%) and $\mathrm{Cl}(0-0.20 \mathrm{wt} . \%)$ content. The micas in Rb-rich Mus- $\mathrm{Ab}$ pegmatite have higher $\mathrm{Rb}_{2} \mathrm{O}$ (2900-4100 ppm). $\mathrm{K} / \mathrm{Rb}$ values of $\mathrm{Rb}$-rich pegmatites range from 26 to 18. According to mica classification in terms of composition and octahedral site occupancy [49], diocthedral micas from barren Mus-Mc-Ab pegmatite are classified mainly as muscovite and ferroan muscovite, whereas micas from Rb-rich Mus-Ab pegmatite are muscovite (Figure 7).

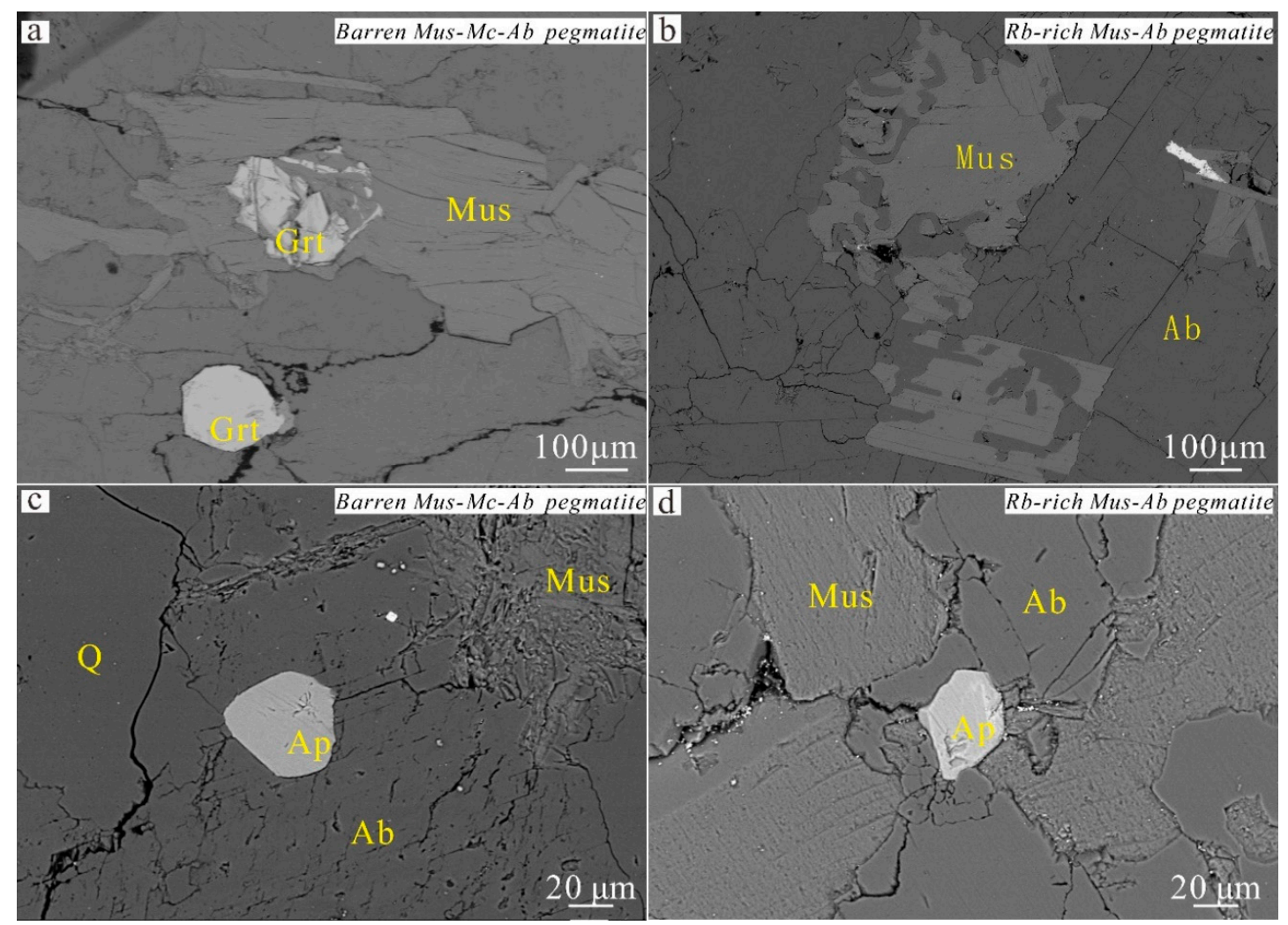

Figure 6. Back-scattered electron (BSE) images of typical muscovite $(\mathbf{a}, \mathbf{b})$ and apatite $(\mathbf{c}, \mathbf{d})$ from the barren Mus-Mc-Ab pegmatites and the Rb-rich Mus-Ab pegmatites. Abbreviation: Mus = muscovite; Grt = garnet; $\mathrm{Q}=$ quartz; $\mathrm{Ab}=$ albite; $\mathrm{Ap}=$ apatite. 
Table 2. Electron microprobe analyses in wt.\% of micas from barren Mus-Mc-Ab and Rb-rich Mus-Ab pegmatite.

\begin{tabular}{|c|c|c|c|c|c|c|c|c|c|c|c|c|c|}
\hline \multirow{2}{*}{ Sample } & \multicolumn{9}{|c|}{ Muscovite from Barren Pegmatite } & \multicolumn{4}{|c|}{ Muscovite from Rb-Rich Pegmatite } \\
\hline & LZP03-1-5 & LZP03-1-6 & LZP03-1-7 & DZXG01-1-4 & DZXG01-1-5 & DZXG01-1-6 & XJ03-1-6 & $\mathrm{XJ} 03-1-7$ & XJ03-1-8 & XJ01-1-12 & XJ01-1-13 & XJ01-1-14 & XJ01-1-15 \\
\hline $\mathrm{SiO}_{2}$ & 45.84 & 45.44 & 45.84 & 45.87 & 45.94 & 45.73 & 47.58 & 47.42 & 48.14 & 48.21 & 48.89 & 46.48 & 46.14 \\
\hline $\mathrm{TiO}_{2}$ & 0.27 & 0.23 & 0.27 & 0.21 & 0.17 & 0.14 & 0.47 & 0.97 & 0.64 & 0.03 & bdl. & bdl. & 0.02 \\
\hline $\mathrm{Al}_{2} \mathrm{O}_{3}$ & 35.14 & 35.37 & 35.43 & 34.06 & 34.30 & 34.33 & 32.78 & 30.1 & 30.52 & 36.48 & 35.16 & 37.18 & 37.17 \\
\hline $\mathrm{FeO}$ & 2.10 & 1.98 & 1.92 & 2.29 & 2.35 & 2.25 & 2.48 & 3.00 & 3.64 & 0.63 & 0.32 & 0.33 & 0.28 \\
\hline $\mathrm{MnO}$ & 0.03 & bdl. & 0.02 & 0.04 & 0.03 & 0.07 & 0.00 & 0.03 & 0.00 & bdl. & 0.08 & 0.02 & bdl. \\
\hline $\mathrm{MgO}$ & 0.67 & 0.71 & 0.64 & 0.57 & 0.55 & 0.61 & 0.54 & 1.62 & 1.10 & 0.02 & bdl. & bdl. & bdl. \\
\hline $\mathrm{CaO}$ & 0.03 & 0.03 & 0.07 & bdl. & bdl. & bdl. & 0.02 & bdl. & 0.06 & 0.02 & 0.01 & 0.03 & bdl. \\
\hline $\mathrm{Na}_{2} \mathrm{O}$ & 0.54 & 0.57 & 0.64 & 0.49 & 0.45 & 0.56 & 0.10 & 0.16 & 0.14 & 0.52 & 0.75 & 0.96 & 0.89 \\
\hline $\mathrm{K}_{2} \mathrm{O}$ & 10.04 & 9.96 & 9.91 & 10.28 & 10.21 & 10.51 & 10.65 & 10.91 & 10.24 & 8.26 & 8.01 & 8.31 & 8.27 \\
\hline $\mathrm{Rb}_{2} \mathrm{O}$ & bdl. & bdl. & bdl. & 0.08 & bdl. & bdl. & bdl. & bdl. & bdl. & 0.29 & 0.41 & 0.41 & 0.38 \\
\hline $\mathrm{Cl}$ & bdl. & bdl. & 0.05 & bdl. & bdl. & bdl. & 0.02 & bdl. & 0.03 & bdl. & 0.02 & bdl. & bdl. \\
\hline $\mathrm{F}$ & bdl. & 0.29 & bdl. & 0.36 & 0.26 & 0.44 & bdl. & 0.2 & 0.26 & 0.05 & 0.13 & 0.08 & 0.01 \\
\hline $\mathrm{Li}_{2} \mathrm{O}^{*}$ & 0.01 & 0.24 & 0.01 & 0.29 & 0.22 & 0.36 & 0.01 & 0.17 & 0.22 & 0.05 & 0.12 & 0.08 & 0.02 \\
\hline $\mathrm{H}_{2} \mathrm{O}^{*}$ & 4.47 & 4.32 & 4.47 & 4.25 & 4.31 & 4.22 & 4.46 & 4.32 & 4.31 & 4.54 & 4.46 & 4.47 & 4.48 \\
\hline $\mathrm{O}=\mathrm{F}, \mathrm{Cl}$ & 0.00 & 0.12 & 0.01 & 0.15 & 0.11 & 0.19 & 0.00 & 0.08 & 0.12 & 0.02 & 0.06 & 0.04 & 0.00 \\
\hline Total & 99.14 & 99.03 & 99.26 & 98.66 & 98.69 & 99.05 & 99.11 & 98.90 & 99.30 & 99.08 & 98.31 & 98.31 & 97.66 \\
\hline $\mathrm{K} / \mathrm{Rb}$ & - & - & - & 117 & - & - & - & - & - & 26 & 18 & 18 & 20 \\
\hline \multicolumn{14}{|c|}{$\mathrm{O}=22$ atoms per formula unit (a.p.f.u.) } \\
\hline $\mathrm{Si}$ & 6.15 & 6.10 & 6.14 & 6.20 & 6.20 & 6.17 & 6.40 & 6.44 & 6.50 & 6.33 & 6.46 & 6.18 & 6.17 \\
\hline $\mathrm{Al}^{\mathrm{IV}}$ & 1.85 & 1.90 & 1.86 & 1.80 & 1.80 & 1.83 & 1.60 & 1.56 & 1.50 & 1.67 & 1.54 & 1.82 & 1.83 \\
\hline $\mathrm{Al}^{\mathrm{VI}}$ & 3.71 & 3.70 & 3.73 & 3.63 & 3.66 & 3.62 & 3.59 & 3.26 & 3.35 & 3.98 & 3.94 & 4.00 & 4.03 \\
\hline $\mathrm{Ti}$ & 0.03 & 0.02 & 0.03 & 0.02 & 0.02 & 0.01 & 0.05 & 0.10 & 0.06 & 0.00 & 0.00 & 0.00 & 0.00 \\
\hline $\mathrm{Fe}$ & 0.24 & 0.22 & 0.21 & 0.26 & 0.27 & 0.25 & 0.28 & 0.34 & 0.41 & 0.07 & 0.04 & 0.04 & 0.03 \\
\hline Mn & 0.00 & 0.00 & 0.00 & 0.00 & 0.00 & 0.01 & 0.00 & 0.00 & 0.00 & 0.00 & 0.01 & 0.00 & 0.00 \\
\hline $\mathrm{Mg}$ & 0.13 & 0.14 & 0.13 & 0.11 & 0.11 & 0.12 & 0.11 & 0.33 & 0.22 & 0.00 & 0.00 & 0.00 & 0.00 \\
\hline $\mathrm{Li}$ & 0.01 & 0.13 & 0.01 & 0.16 & 0.12 & 0.19 & 0.01 & 0.09 & 0.12 & 0.03 & 0.06 & 0.04 & 0.01 \\
\hline $\mathrm{Ca}$ & 0.00 & 0.00 & 0.01 & 0.00 & 0.00 & 0.00 & 0.00 & 0.00 & 0.01 & 0.00 & 0.00 & 0.00 & 0.00 \\
\hline $\mathrm{Na}$ & 0.14 & 0.15 & 0.17 & 0.13 & 0.12 & 0.15 & 0.03 & 0.04 & 0.04 & 0.13 & 0.19 & 0.25 & 0.23 \\
\hline $\mathrm{K}$ & 1.72 & 1.71 & 1.69 & 1.77 & 1.76 & 1.81 & 1.83 & 1.89 & 1.76 & 1.38 & 1.35 & 1.41 & 1.41 \\
\hline $\mathrm{Rb}$ & 0.00 & 0.00 & 0.00 & 0.01 & 0.00 & 0.00 & 0.00 & 0.00 & 0.00 & 0.02 & 0.03 & 0.04 & 0.03 \\
\hline $\mathrm{F}$ & 0.00 & 0.12 & 0.00 & 0.15 & 0.11 & 0.19 & 0.00 & 0.09 & 0.11 & 0.02 & 0.06 & 0.04 & 0.00 \\
\hline $\mathrm{Cl}$ & 0.00 & 0.00 & 0.01 & 0.00 & 0.00 & 0.00 & 0.00 & 0.00 & 0.01 & 0.00 & 0.00 & 0.00 & 0.00 \\
\hline $\mathrm{OH}^{*}$ & 4.00 & 3.88 & 3.99 & 3.85 & 3.89 & 3.81 & 4.00 & 3.91 & 3.88 & 3.98 & 3.94 & 3.96 & 4.00 \\
\hline
\end{tabular}

Notes: $\mathrm{Li}_{2} \mathrm{O}$ calculated after Van Lichtervelde et al. (2008) [50]; $\mathrm{H}_{2} \mathrm{O}$ calculated after Tindle and Webb (1990) [51]; * means values calculated by stoichiometry; bdl. means below the detection limit. 


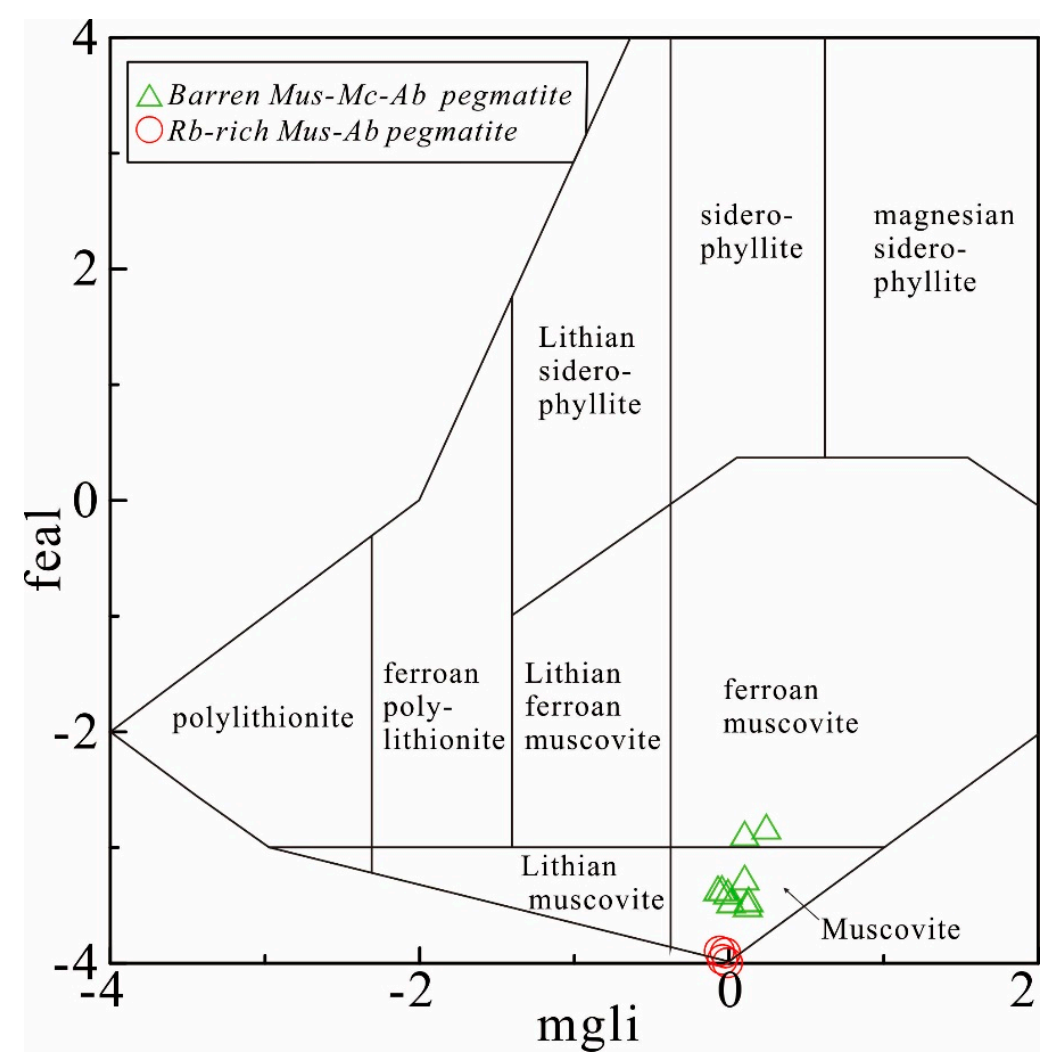

Figure 7. Classification diagram for trioctahedral and dioctahedral micas after Tischendorf et al. (1997) [49]. Micas from the barren Mus-Mc-Ab and Rb-rich Mus-Ab pegmatite are plotted. mgli = Mg-Li, $\mathrm{feal}=\mathrm{Fe}+\mathrm{Mn}+\mathrm{Ti}-\mathrm{Al}^{\mathrm{VI}}$.

Composition of K-feldspar and albite from the barren Mus-Mc-Ab pegmatite and albite from $\mathrm{Rb}$-rich Mus-Ab pegmatite are presented in Table 3. The orthoclase component of the K-feldspar ranges between $\mathrm{Or}_{92}$ and $\mathrm{Or}_{96}$ from the barren Mus-Mc-Ab pegmatite. K-feldspar is absent from the Rb-rich Mus-Ab pegmatite. The albite component of the albite from barren Mus-Mc-Ab pegmatite ranges between $\mathrm{Ab}_{89}$ and $\mathrm{Ab}_{98}$, whereas that of the albite from $\mathrm{Rb}$-rich Mus- $\mathrm{Ab}$ pegmatite ranges between $\mathrm{Ab}_{99}$ and $\mathrm{Ab}_{100}$. The $\mathrm{Rb}_{2} \mathrm{O}$ content was detected (0-1000 ppm) in K-feldspar from Mus-Mc-Ab barren pegmatite. Albite from $\mathrm{Rb}$-rich Mus- $\mathrm{Ab}$ pegmatite all contain $\mathrm{Rb}_{2} \mathrm{O}$ content, ranging from 1000 to 1400 ppm. 
Table 3. Electron microprobe analyses in wt.\% of feldspars from barren Mus-Mc-Ab and Rb-rich Mus-Ab pegmatite.

\begin{tabular}{|c|c|c|c|c|c|c|c|c|c|c|c|c|c|c|c|c|}
\hline \multirow{2}{*}{ Sample } & \multicolumn{5}{|c|}{ Albite from Barren Pegmatite } & \multicolumn{5}{|c|}{ K-Feldspar from Barren Pegmatite } & \multicolumn{6}{|c|}{ Albite from Rb-Rich Pegmatite } \\
\hline & DZXG01-1 & DZXG01-2 & DZXG01-3 & DZXG01-4 & $\mathrm{XJ} 03-2$ & DZXG01-1 & DZXG01-2 & DZXG01-3 & DZXG01-4 & $\mathrm{XJ03-3}$ & $\mathrm{XJ01-2}$ & $\mathrm{XJ01-3}$ & $\mathrm{XJ01-4}$ & XJ01-5 & XJ01-6 & $\mathrm{XJ01-8}$ \\
\hline $\mathrm{SiO}_{2}$ & 65.61 & 67.85 & 66.14 & 65.95 & 67.97 & 64.62 & 64.12 & 64.45 & 64.35 & 64.68 & 69.01 & 69.45 & 68.31 & 67.81 & 68.18 & 68.22 \\
\hline $\mathrm{Al}_{2} \mathrm{O}_{3}$ & 20.39 & 19.13 & 19.93 & 20.08 & 19.25 & 17.86 & 17.86 & 18.11 & 17.90 & 17.98 & 19.80 & 19.62 & 19.53 & 19.52 & 19.10 & 19.30 \\
\hline $\mathrm{CaO}$ & 2.10 & 0.44 & 1.98 & 2.01 & 0.35 & 0.03 & 0.02 & 0.03 & 0.02 & 0.06 & 0.09 & 0.04 & 0.05 & 0.03 & bdl. & 0.02 \\
\hline $\mathrm{Na}_{2} \mathrm{O}$ & 10.51 & 11.37 & 10.71 & 10.79 & 11.62 & 0.55 & 0.44 & 0.81 & 0.62 & 0.90 & 11.30 & 11.30 & 11.17 & 11.49 & 11.41 & 11.57 \\
\hline $\mathrm{K}_{2} \mathrm{O}$ & 0.27 & 0.09 & 0.19 & 0.21 & 0.12 & 16.20 & 16.47 & 15.71 & 15.82 & 15.99 & 0.06 & 0.04 & 0.08 & 0.09 & 0.11 & 0.14 \\
\hline $\mathrm{P}_{2} \mathrm{O}_{5}$ & 0.14 & 0.13 & 0.11 & 0.18 & 0.05 & 0.06 & 0.09 & 0.12 & 0.05 & 0.09 & 0.45 & 0.04 & 0.08 & 0.22 & 0.14 & 0.28 \\
\hline $\mathrm{Rb}_{2} \mathrm{O}$ & bdl. & bdl. & bdl. & bdl. & bdl. & 0.06 & 0.10 & bdl. & bdl. & bdl. & 0.12 & 0.10 & 0.12 & 0.13 & 0.14 & 0.10 \\
\hline Total & 99.02 & 99.01 & 99.06 & 99.22 & 99.36 & 99.38 & 99.10 & 99.23 & 98.76 & 99.70 & 100.84 & 100.58 & 99.33 & 99.28 & 99.07 & 99.63 \\
\hline \multicolumn{17}{|c|}{$\mathrm{O}=8$ atoms per formula unit (a.p.f.u.) } \\
\hline $\mathrm{Si}$ & 2.92 & 3.00 & 2.94 & 2.93 & 2.99 & 3.01 & 3.00 & 3.00 & 3.01 & 3.00 & 2.99 & 3.01 & 3.00 & 2.99 & 3.01 & 3.00 \\
\hline $\mathrm{Al}$ & 1.07 & 1.00 & 1.04 & 1.05 & 0.99 & 0.98 & 0.99 & 0.99 & 0.99 & 0.98 & 1.01 & 1.00 & 1.01 & 1.01 & 0.99 & 1.00 \\
\hline $\mathrm{Ca}$ & 0.10 & 0.02 & 0.09 & 0.10 & 0.01 & 0.00 & 0.00 & 0.00 & 0.00 & 0.00 & 0.00 & 0.00 & 0.00 & 0.00 & 0.00 & 0.00 \\
\hline $\mathrm{Na}$ & 0.91 & 0.97 & 0.92 & 0.93 & 0.99 & 0.05 & 0.04 & 0.07 & 0.06 & 0.08 & 0.95 & 0.95 & 0.95 & 0.98 & 0.98 & 0.98 \\
\hline $\mathrm{K}$ & 0.02 & 0.01 & 0.01 & 0.01 & 0.00 & 0.96 & 0.98 & 0.93 & 0.94 & 0.94 & 0.00 & 0.00 & 0.00 & 0.00 & 0.01 & 0.01 \\
\hline $\mathrm{P}$ & 0.00 & 0.00 & 0.00 & 0.00 & 0.00 & 0.00 & 0.00 & 0.00 & 0.00 & 0.00 & 0.01 & 0.00 & 0.00 & 0.00 & 0.00 & 0.01 \\
\hline $\mathrm{Rb}$ & 0.00 & 0.00 & 0.00 & 0.00 & 0.00 & 0.00 & 0.00 & 0.00 & 0.00 & 0.00 & 0.00 & 0.00 & 0.00 & 0.00 & 0.00 & 0.00 \\
\hline \multicolumn{17}{|c|}{ End-member components (moles \%) } \\
\hline An & 9.80 & 2.10 & 9.20 & 9.20 & 1.60 & 0.20 & 0.10 & 0.20 & 0.10 & 0.30 & 0.43 & 0.17 & 0.26 & 0.16 & 0.00 & 0.10 \\
\hline $\mathrm{Ab}$ & 88.70 & 97.40 & 89.80 & 89.60 & 97.70 & 4.90 & 3.90 & 7.30 & 5.60 & 7.90 & 99.21 & 99.63 & 99.29 & 99.34 & 99.39 & 99.11 \\
\hline Or & 1.50 & 0.50 & 1.10 & 1.20 & 0.70 & 95.00 & 96.00 & 92.60 & 94.30 & 91.90 & 0.36 & 0.20 & 0.45 & 0.50 & 0.61 & 0.79 \\
\hline
\end{tabular}

Notes: bdl. means below the detection limit. 
Most apatite grains from the studied samples are euhedral, and no prominent zonation was observed (Figure $6 \mathrm{c}, \mathrm{d}$ ), indicating a magmatic origin. The analytical data of apatite composition are summarized in Table 4 and Figure 8 . The $\mathrm{P}_{2} \mathrm{O}_{5}$ contents of the apatite from barren Mus-Mc-Ab pegmatite (41.47-42.20 wt.\%) are more or less the same with those from Rb-rich Mus-Ab pegmatite (41.12-42.83 wt.\%) (Figure 8a), whereas the $\mathrm{CaO}$ contents of the apatite from the barren Mus-Mc-Ab pegmatite (54.39-55.18 wt.\%) are higher than those of the apatite from Rb-rich Mus-Ab pegmatite (36.29-40.17 wt.\%) (Figure 8b). Moreover, the MnO contents of the apatite from the Rb-rich Mus- $\mathrm{Ab}$ pegmatite (14.51-19.12 wt.\%) are much higher than those of the apatite from barren Mus-Mc-Ab pegmatite (0.34-0.54 wt.\%) (Figure 8c). All of the apatite grains analyzed have high fluorine contents (2.85-3.56 wt.\%) (Table 4), classifying them as fluorapatite. However, fluorine contents were indistinguishable between barren Mus-Mc-Ab and Rb-rich Mus-Ab pegmatite (Figure 8d). In contrast, chlorine contents are lower and are well distinguished between barren Mus-Mc-Ab and $\mathrm{Rb}$-rich Mus- $\mathrm{Ab}$ pegmatite, with the latter displaying higher $\mathrm{Cl}$ contents (1200-1600 ppm) (Figure 8d). Furthermore, apatite $\mathrm{F} / \mathrm{Cl}$ ratios of $\mathrm{Rb}$-rich Mus-Ab pegmatite (20-29) are much lower than those of barren Mus-Mc-Ab pegmatite (164-169) (Figure 8f).
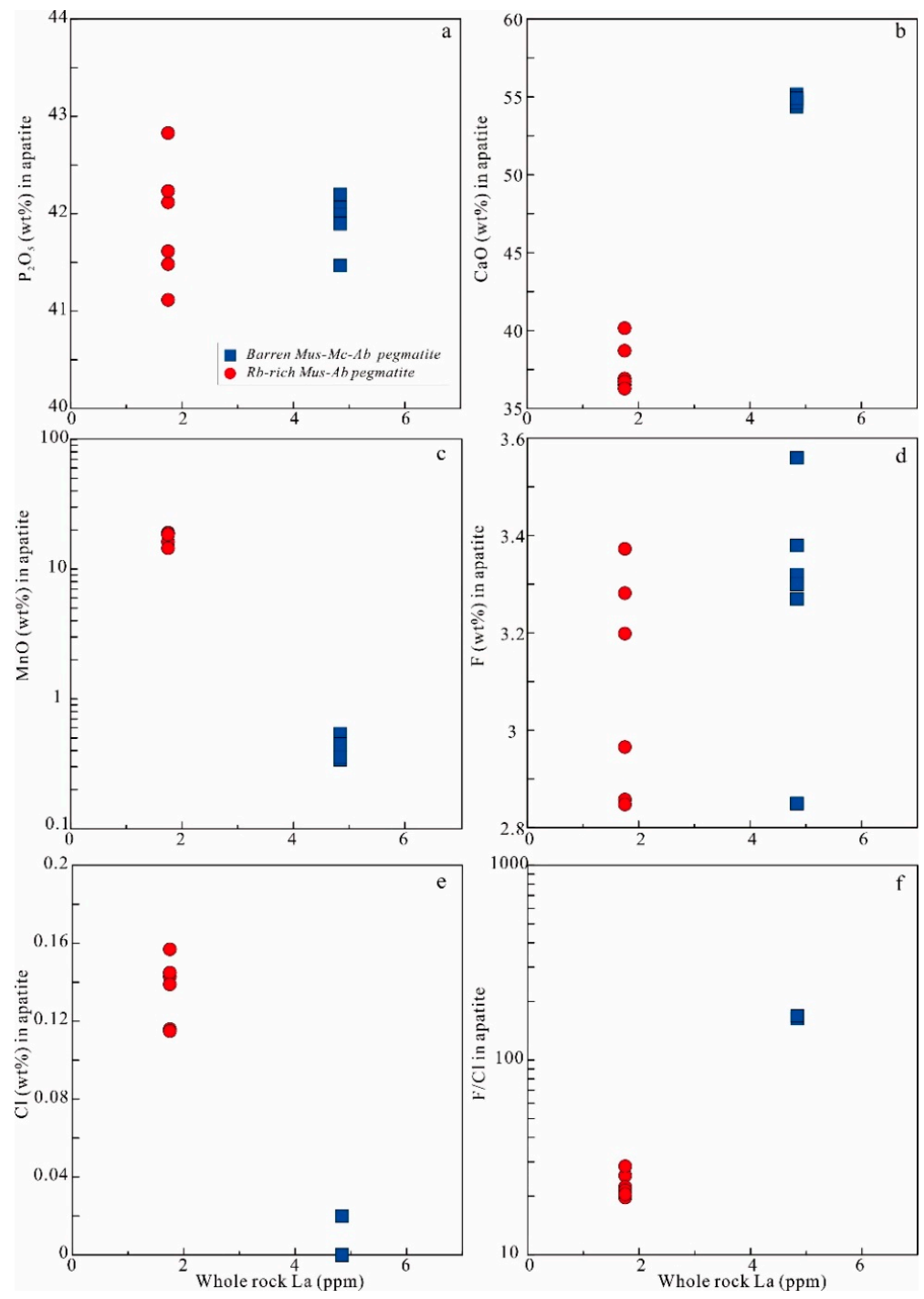

Figure 8. (a-f) correlation between major elements in apatite and the whole-rock La content. Note that whole-rock La contents are from the average values of the corresponding pegmatites. 
Table 4. Electron microprobe analyses in wt.\% of apatites from barren Mus-Mc-Ab and Rb-rich Mus-Ab pegmatite.

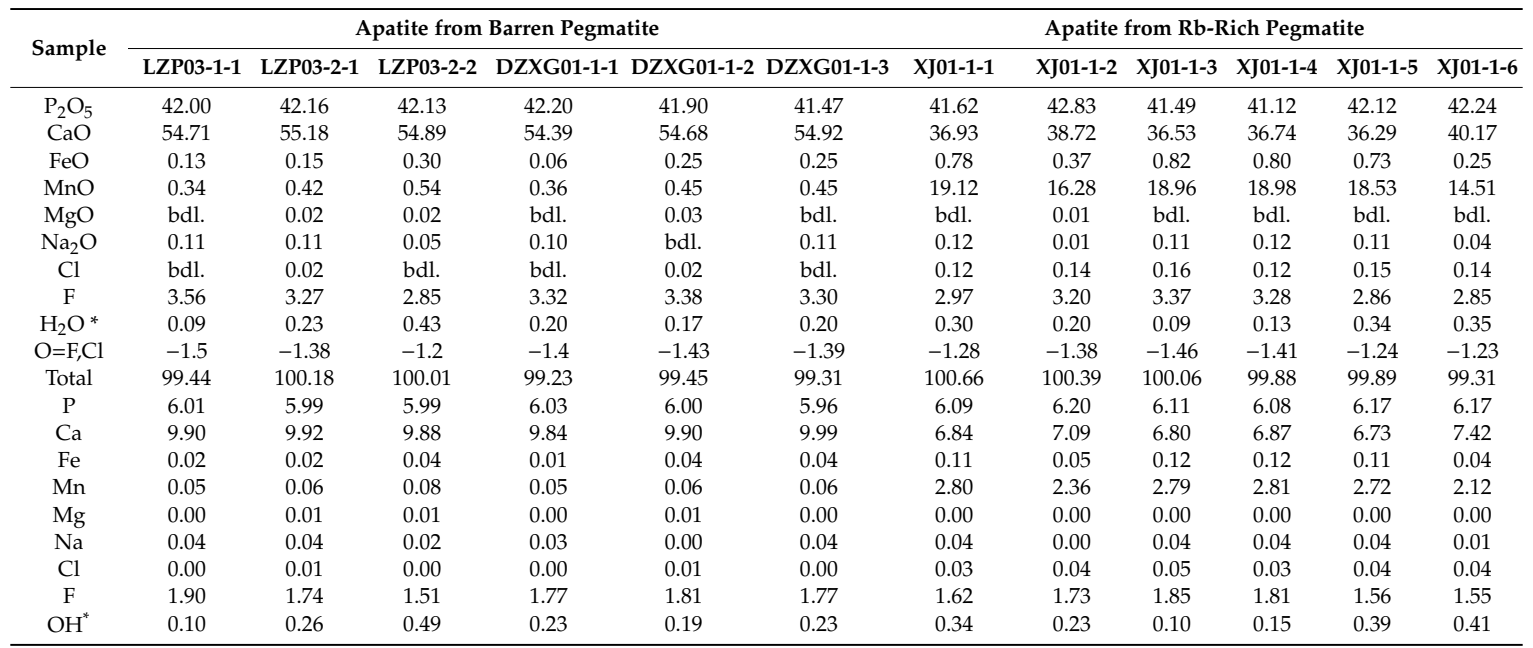

Notes: * means values calculated by stoichiometry; bdl. means below the detection limit.

\subsection{Zircon $\mathrm{U}-\mathrm{Pb}$ Ages}

Zircon crystals from barren Mus-Mc-Ab pegmatite (LZP03 and DZXG01) and Rb-rich Mus-Ab pegmatite (XJ01-4) samples display variable sizes (e.g., LZP03: 100-200 $\mu \mathrm{m}$; DZXG01: 50-120 $\mu \mathrm{m}$; XJ01-4: 100-300 $\mu \mathrm{m}$ ) (Figure 9). The U-Pb dating data are shown in Table 5. The plotted zircon U-Pb ages are shown in Figure 10.

Table 5. Result of LA-ICP-MS zircon U-Pb dating of the Lizhaping and Dazengxigou barren pegmatites and Xinjian Rb-rich pegmatites.

\begin{tabular}{|c|c|c|c|c|c|c|c|c|c|c|c|}
\hline \multirow{2}{*}{ SampleTitle } & \multicolumn{2}{|c|}{ Element (ppm) } & \multirow{2}{*}{$\mathrm{Th} / \mathrm{U}$} & \multicolumn{4}{|c|}{ Isotope Ratio } & \multicolumn{4}{|c|}{ Age (Ma) } \\
\hline & Th & $\mathbf{U}$ & & ${ }^{207} \mathrm{~Pb} /{ }^{235} \mathrm{U}$ & $1 \sigma$ & ${ }^{206} \mathrm{~Pb} /{ }^{238} \mathrm{U}$ & $1 \sigma$ & ${ }^{207} \mathrm{~Pb} /{ }^{235} \mathrm{U}$ & $1 \sigma$ & ${ }^{206} \mathrm{~Pb} /{ }^{238} \mathrm{U}$ & 10 \\
\hline \multicolumn{12}{|l|}{ Sample LZP03 } \\
\hline 1 & 816 & 586 & 1.393 & 1.1429 & 0.0247 & 0.1270 & 0.0018 & 774 & 12 & 771 & 10 \\
\hline 2 & 1458 & 1114 & 1.308 & 0.5944 & 0.0150 & 0.0760 & 0.0013 & 474 & 10 & 472 & 8 \\
\hline 3 & 1089 & 888 & 1.226 & 0.7227 & 0.0210 & 0.0916 & 0.0017 & 552 & 12 & 565 & 10 \\
\hline 4 & 1259 & 2493 & 0.505 & 0.2266 & 0.0060 & 0.0327 & 0.0006 & 207 & 5 & 208 & 4 \\
\hline 5 & 1179 & 1679 & 0.702 & 0.9216 & 0.0163 & 0.1084 & 0.0015 & 663 & 9 & 664 & 9 \\
\hline 6 & 771 & 649 & 1.189 & 1.2463 & 0.0303 & 0.1375 & 0.0025 & 822 & 14 & 831 & 14 \\
\hline 7 & 491 & 2275 & 0.216 & 0.2310 & 0.0060 & 0.0334 & 0.0005 & 211 & 5 & 212 & 3 \\
\hline 8 & 1868 & 727 & 2.569 & 1.0417 & 0.0214 & 0.1174 & 0.0017 & 725 & 11 & 716 & 10 \\
\hline \multicolumn{12}{|l|}{ Sample DZXG01 } \\
\hline 1 & 218 & 10,836 & 0.020 & 0.3170 & 0.0067 & 0.0355 & 0.0006 & 280 & 5 & 225 & 4 \\
\hline 2 & 491 & 7240 & 0.068 & 0.6655 & 0.0283 & 0.0361 & 0.0009 & 518 & 17 & 229 & 5 \\
\hline 3 & 793 & 9015 & 0.088 & 0.4844 & 0.0338 & 0.0362 & 0.0010 & 401 & 23 & 230 & 6 \\
\hline 4 & 186 & 12,941 & 0.014 & 0.5848 & 0.0561 & 0.0411 & 0.0011 & 468 & 36 & 260 & 7 \\
\hline 5 & 1115 & 4882 & 0.228 & 0.5825 & 0.0250 & 0.0354 & 0.0006 & 466 & 16 & 225 & 4 \\
\hline 6 & 323 & 8437 & 0.038 & 1.6545 & 0.0509 & 0.0519 & 0.0011 & 991 & 19 & 326 & 7 \\
\hline 7 & 1358 & 5266 & 0.258 & 0.7202 & 0.0388 & 0.0369 & 0.0007 & 551 & 23 & 234 & 4 \\
\hline 8 & 179 & 6978 & 0.026 & 0.3182 & 0.0092 & 0.0342 & 0.0007 & 281 & 7 & 217 & 4 \\
\hline 9 & 758 & 10,702 & 0.071 & 1.2831 & 0.1187 & 0.0466 & 0.0010 & 838 & 53 & 294 & 6 \\
\hline \multicolumn{12}{|l|}{ Sample XJ01-4 } \\
\hline 1 & 1863 & 1674 & 1.113 & 0.2292 & 0.0079 & 0.0316 & 0.0008 & 210 & 7 & 200 & 5 \\
\hline 2 & 1485 & 1321 & 1.124 & 0.2299 & 0.0079 & 0.0313 & 0.0005 & 210 & 7 & 199 & 3 \\
\hline 3 & 1816 & 1405 & 1.292 & 0.2237 & 0.0056 & 0.0315 & 0.0004 & 205 & 5 & 200 & 2 \\
\hline 4 & 806 & 1094 & 0.737 & 0.2260 & 0.0089 & 0.0333 & 0.0008 & 207 & 7 & 211 & 5 \\
\hline 5 & 542 & 771 & 0.703 & 0.2238 & 0.0087 & 0.327 & 0.0006 & 205 & 7 & 208 & 4 \\
\hline 6 & 446 & 700 & 0.637 & 0.2127 & 0.0091 & 0.0320 & 0.0006 & 196 & 8 & 203 & 4 \\
\hline 7 & 295 & 501 & 0.589 & 0.2246 & 0.0095 & 0.0329 & 0.0006 & 206 & 8 & 209 & 4 \\
\hline
\end{tabular}

Most zircons separated from the sample LZP03 display oscillatory zoning, as shown in the cathodoluminescence (CL) images (Figure 9a), which indicates a magmatic origin. We obtained analyses of eight zircons from sample LZP03 (Figure 10a). The U concentrations of the analyzed zircons 
range from 586 to $2493 \mathrm{ppm}$, Th contents range from 491 to $1868 \mathrm{ppm}$, and the Th/U ratios range from 0.216 to 2.569 . All eight analyses give apparent ${ }^{206} \mathrm{~Pb} /{ }^{238} \mathrm{U}$ ages between $208 \mathrm{Ma}$ and $831 \mathrm{Ma}$, with analyses spots \#4 and \#7 yielding apparent ${ }^{206} \mathrm{~Pb} /{ }^{238} \mathrm{U}$ ages of $208 \pm 4$ and $212 \pm 3 \mathrm{Ma}$, respectively (Figure 10a), which is considered the crystallization age of these pegmatites. The remaining six analyses yielded apparent ${ }^{206} \mathrm{~Pb} /{ }^{238} \mathrm{U}$ ages of $472-831 \mathrm{Ma}$, which were obviously older than the analyses spots \#4 and \#7 (Figure 10a), suggesting that these older zircons had been inherited.

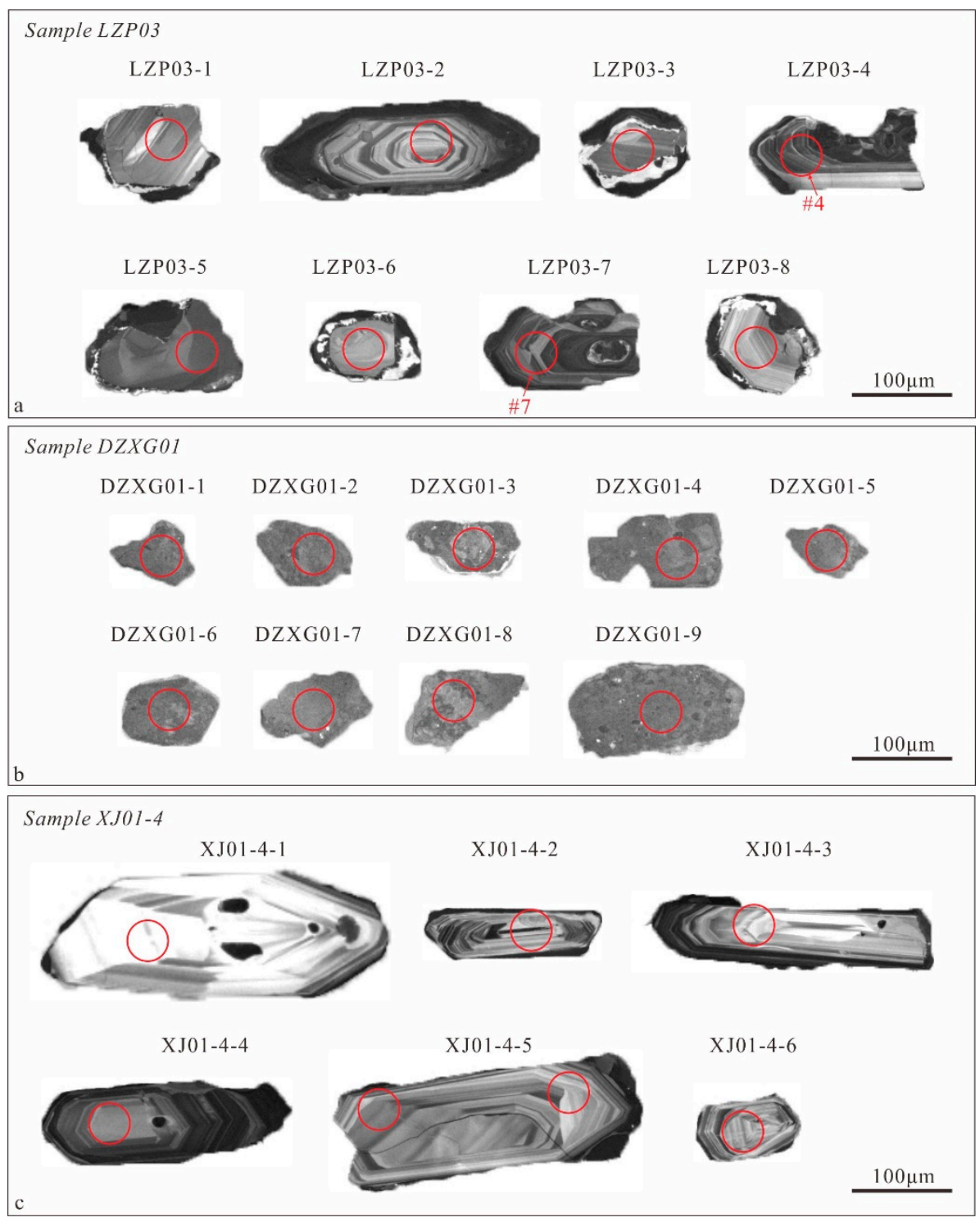

Figure 9. Cathodoluminescence (CL) images of zircons, showing the laser analytic spots, mineral inclusions, and ${ }^{206} \mathrm{~Pb} /{ }^{238} \mathrm{U}$ ages. (a-c) zircons from sample LZP03, DZXG01 and XJ01-4, respectively.

Zircons separated from sample DZXG01 show spongy and porous features on CL images (Figure 9b), suggesting that they have been subjected to metamictization or recrystallization, likely ascribable to high U concentrations or deuteric fluid [52-54]. These features are commonly seen in rare-element pegmatites from other places because of high fluxes and the presence of late-stage hydrothermal fluids [24,25,55-57]. We obtained the analyses of nine zircons from sample DZXG01 (Figure 10b). The zircons analyzed have variable U concentrations (4882-12,941 ppm), Th contents (4179-1358 ppm), and Th/U ratios (0.014-0.258). The ${ }^{206} \mathrm{~Pb} /{ }^{238} \mathrm{U}$ ratios for these nine spots yielded a lower intersection point age of $206 \pm 18 \mathrm{Ma}$, with a MSWD of 9.9 (Figure 10b). 
Zircons separated from the sample XJ01-4 display oscillatory zoning, as shown in the cathodoluminescence (CL) images (Figure 9c), indicating a magmatic origin. We obtained a total of seven analyses of six zircons from sample XJ01-4 (Figure 10c). The analyzed zircons have U and Th concentrations ranging from 501 to $1674 \mathrm{ppm}$, and 295 to $1863 \mathrm{ppm}$. The zircons have high Th/U ratios ranging, from 0.589 to 1.292 (with an average of 0.89), indicative of a magmatic origin [58]. $\mathrm{The}{ }^{206} \mathrm{~Pb} /{ }^{238} \mathrm{U}$ ratios for these seven spots yielded a weighted mean age of $202.9 \pm 4.2 \mathrm{Ma}$, with a mean square weighted deviation (MSWD) of 1.7 (Figure 10c).
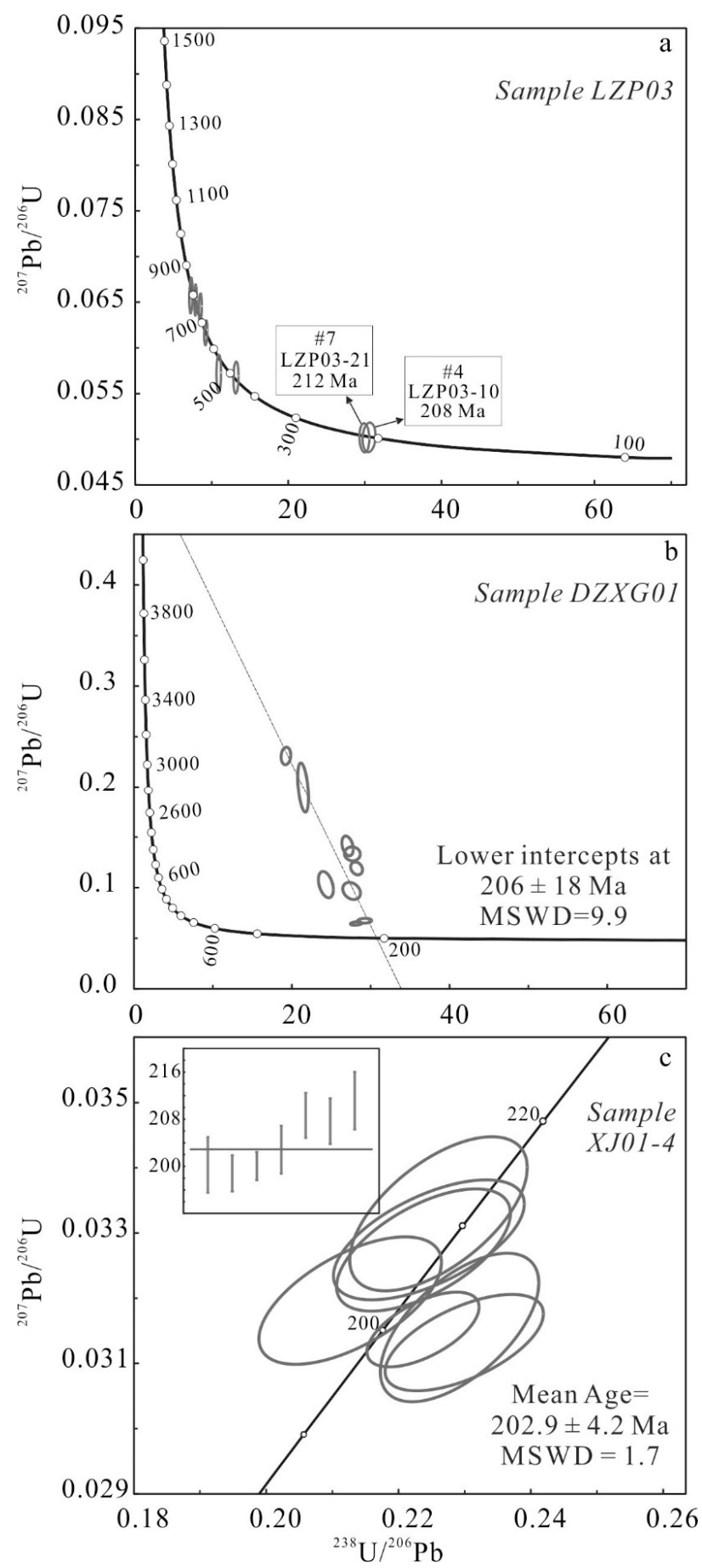

Figure 10. Concordia and weighted average diagrams showing the LA-ICP-MS U-Pb ages of zircons from sample LZP03 (a), DZXG01 (b), and XJ01-4 (c). 


\section{Discussion}

\subsection{Age of Pegmatites}

Although zircon $\mathrm{U}-\mathrm{Pb}$ dating for granitic pegmatites has a few problems, including huge amounts of inherited zircons, or strongly metamicted zircons, the $\mathrm{U}-\mathrm{Pb}$ zircon geochronometer is one of the most important dating methods for rare metal-rich pegmatite, as the closure temperature of the zircon $\mathrm{U}-\mathrm{Pb}$ system is close to the temperature of the magmatic stage of pegmatites [24,25,55-60]. In this study, two spot ages of $208 \pm 4$ and $212 \pm 3$ Ma for the Lizhaping barren pegmatite (sample LZP03), and a lower intersection age of $206 \mathrm{Ma}$ for the Dazengxigou barren pegmatite (sample DZXG01) were determined, as well as a weighted mean age of $202.9 \pm 4.2 \mathrm{Ma}$ for the Xinjian Rb-rich pegmatite (sample XJ01-4) (Figure 10a-c). We interpreted these ages (212-203 Ma) as the emplacement ages of the pegmatites. These results are similar to those of previous $\mathrm{U}-\mathrm{Pb}$ dating studies that demonstrated the existence of Late Triassic magmatic activity in the middle segment of SQB [39,47,48,61-65].

\subsection{Relationship between the Pegmatites and Granites}

Triassic Granitic plutons are widespread in the Foping-Ningshan area in the middle segment of SQB. Geochronological data indicate that these plutons were emplaced during the Triassic (230-200 Ma) [39,47,48,61-65]. These plutons include Xiba, Huayang, Wulong, Qinlingliang, Xichahe, Laocheng, and Yanzhiba plutons (Figure 1b), which consist of diorite, granodiorite, monzogranite, and biotite granite.

Granitic pegmatite is generally considered a product of volatile-rich (e.g., B, F, P) residual magma through the differentiation of parental granitic magma $[6,7,9]$. The pegmatite field surrounding a source granite is generally zoned with respect to the distribution of rare elements [1,2,4]. As emphasized by Dill (2015) [66], the temporal relation between pegmatites and neighboring granites plays a key role in distinguishing the origin of pegmatites. According to Selway et al. (2005) [67], pegmatite dikes with the most economic potential for Li-Cs-Ta deposits occur the greatest distance (up to $10 \mathrm{~km}$ ) from the parent granite. In our study area, pegmatites are juxtaposed with the Yanzhiba pluton (Figure 1c). Some of the pegmatites intruded into the monzogranites, with a progressive evolution contact (Figure 2a). The Yanzhiba pluton can be divided into Yanzhiba granodiorites [48], Yanzhiba two-mica monzogranites [47], and Lanbandeng two-mica monzogranites [48]. Previous chronological study has shown that the Yanzhiba granodiorite formed at $220 \mathrm{Ma}$ [48], and the two-mica monzogranites in Yanzhiba and the Lanbandeng intrusion formed at $210 \mathrm{Ma}$ [47,48]. As mentioned above, the pegmatites formed in the Late Triassic (212-203 Ma), slightly younger than the two-mica monzogranites in Yanzhiba and the Lanbandeng intrusion. The spatial and temporal distribution of pegmatites and granites reveals that the Yanzhiba and Lanbandeng two-mica monzogranites are the potential parental granite of these pegmatites.

For geochemical comparison, we collected data of the Yanzhiba and Lanbandeng two-mica monzogranites published by Jiang et al. (2010) and Yang et al. (2012) [47,48]. The $\mathrm{Zr} / \mathrm{Hf}, \mathrm{Rb} / \mathrm{Sr}$ or $\mathrm{Nb} / \mathrm{Ta}$ ratios are used generally as an indicator of pegmatite evolution [8,68-75]. The trends of the plots of La versus $\mathrm{Zr} / \mathrm{Hf}$ ratios (Figure 11a) could be interpreted in terms of zircon fractional crystallization from monzogranites and two-mica monzogranites to the pegmatites. The plots of La versus $\mathrm{Rb} / \mathrm{Sr}$ ratios (Figure 11b) show evolution trends that could be interpreted in terms of feldspar and mica (biotite and/or muscovite) and fractional crystallization. The trends of the plots of La versus $\mathrm{Nb} / \mathrm{Ta}$ ratios could be interpreted in terms of mica (biotite and/or muscovite) fractional crystallization (Figure 11c). The reasons for these hypotheses are as follows: (1) Previous experimental studies have shown that the ratio $\mathrm{D}_{\mathrm{Hf}} / \mathrm{D}_{\mathrm{Zr}}$ of the partition coefficients of $\mathrm{Zr}$ and Hf between zircon and melt are low (0.5 to 0.2$)$ for metaluminous and peraluminous melts [72]. Consequently, zircon fractionation strongly decreases $\mathrm{Zr} / \mathrm{Hf}$ in some peraluminous granites [8,70,71,74]. (2) $\mathrm{Sr}$ is more compatible in feldspar, whereas $\mathrm{Rb}$ is more compatible in mica. In response to feldspar fractionation and mica concentration, there is a marked depletion of $\mathrm{Sr}$ and enrichment of $\mathrm{Rb}$, resulting in a high $\mathrm{Rb} / \mathrm{Sr}$ 
ratio [68,69]. (3) Experimental studies have shown that $\mathrm{Nb}$ preferentially partitions into mica relative to Ta in the granitic system [75]. Thus, Ta concentrates in residual melt relative to $\mathrm{Nb}$. As a result, the residual melt becomes enriched in Ta relative to $\mathrm{Nb}$ and yields lower $\mathrm{Nb} / \mathrm{Ta}$ ratios. Accordingly, the barren and $\mathrm{Rb}$-rich pegmatites in the study area are both derived from the fractionation of Yanzhiba and Lanbandeng two-mica monzogranites.
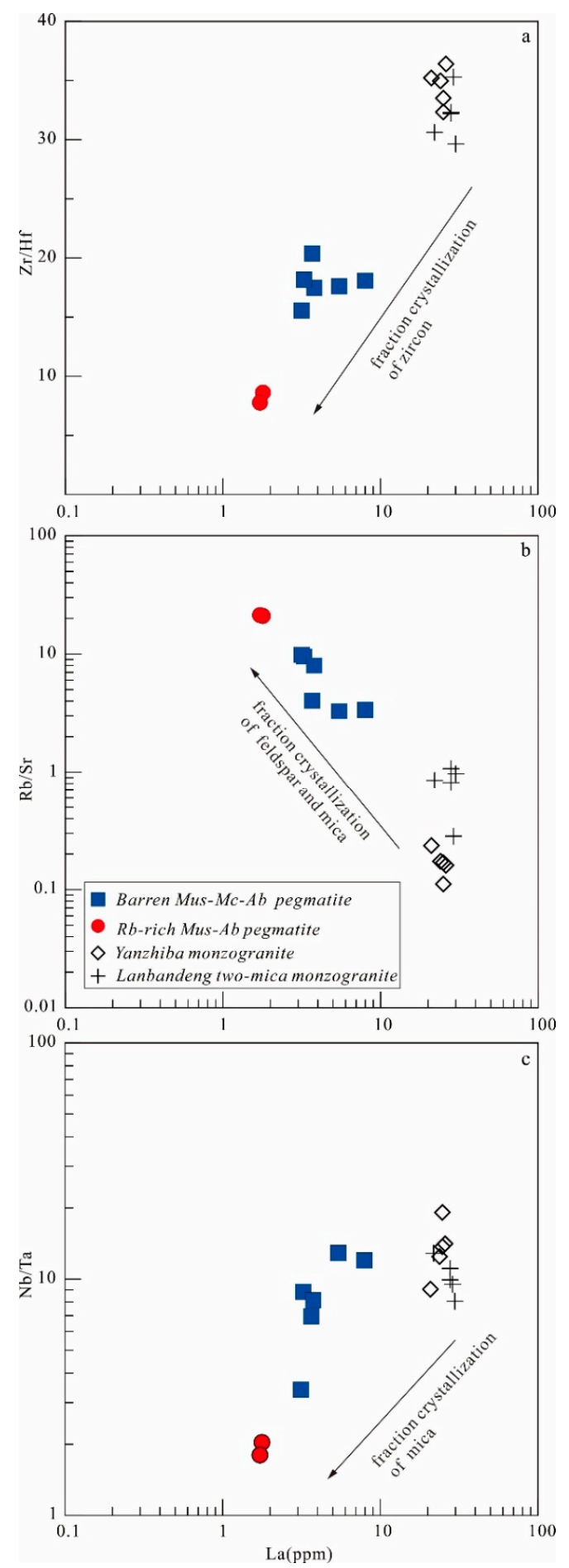

Figure 11. Variations of La versus (a) $\mathrm{Zr} / \mathrm{Hf}$, (b) $\mathrm{Rb} / \mathrm{Sr}$ and (c) $\mathrm{Nb} / \mathrm{Ta}$ for whole-rock samples. A fractional crystallization trend is shown (see text for details). Literature data of Yanzhiba and Lanbandeng two-mica monzogranites are from Jiang et al., 2010; Yang et al., 2012 [47,48]. 
This hypothesis is supported also by other geochemical signatures. In the chondrite-normalized REE pattern (Figure 5a), the barren pegmatites show a seagull-like normalized REE pattern, with a depleted LREE and a pronounced negative Eu anomaly. In contrast, Yanzhiba and Lanbandeng two-mica monzogranites show a right-declined pattern with a weak Eu anomaly. Those negative Eu anomalies are more pronounced in pegmatite than in two-mica monzogranites can be interpreted as being caused by feldspar fractionation during granite emplacement because Eu prefers to partition into plagioclase. The depleted LREE pattern was caused by accessory minerals, considering that LREE are incorporated generally in the early-crystallizing accessory minerals such as monazite (80-85 wt.\%) and apatite ( $10 \mathrm{wt} . \%)$, which is virtually devoid in the last solidified pegmatites [76,77]. Rb-rich Mus- $\mathrm{Ab}$ pegmatite have a significantly different REE pattern, with much lower $\sum$ REE concentrations and the lack of an Eu anomaly. Fractionation of the accessory minerals (i.e., apatite and monazite) in parental granite can cause the depletion of LREE in the residual magma, and fractionation of garnet result in the depletion HREE. As for the lack of an Eu anomaly, this phenomenon can be also observed in some other rare metal-bearing pegmatites $[8,78,79]$. Previous study pointed out that oxidation of $\mathrm{Eu}^{2+}$ to $\mathrm{Eu}^{3+}$ can make it less compatible in the Ca sites of plagioclase [80-82]. The REE data for plagioclase show a decrease of the positive Eu-anomaly and then a change to negative Eu-anomaly from the wall and intermediate zone to the more evolved inner zones $[83,84]$. Thus, the absence of an $\mathrm{Eu}$ anomaly can be attributed to the elevated oxidation state during the pegmatite evolution. Evidence for the oxidizing conditions during the formation of $\mathrm{Rb}$-rich Mus-Ab pegmatite is recorded in the abundance of cassiterite and columbite-group mineral. On the primitive mantle normalized spider diagram, compared with granite, the pegmatites show positive in $\mathrm{Rb}, \mathrm{U}$, and $\mathrm{P}$ anomalies, and negative anomalies of $\mathrm{Ba}, \mathrm{Sr}, \mathrm{Zr}$, and Ti (Figure $5 \mathrm{~b}$ ). The positive in $\mathrm{Rb}, \mathrm{U}$, and $\mathrm{P}$ anomalies in the pegmatites is ascribed to their high incompatible properties, i.e., they do not favor crystallization of their individual (accessory) minerals at the early magmatic stage [85-88]. The depletion in $\mathrm{Ba}$ and $\mathrm{Sr}$ in the pegmatite can be ascribed to feldspar fractionation in the two-mica monzogranites [89], whereas depletion in $\mathrm{Ti}$ and $\mathrm{Zr}$ is the result of early-crystallized ferromagnesian minerals (biotite and Fe-Ti oxides) fractionation and zircon fractionation, respectively [77,90]. The trace element abundance diagrams of the Rb-rich pegmatites are characterized by pronounced enrichment in $\mathrm{Rb}, \mathrm{Nb}, \mathrm{P}, \mathrm{Sr}$, and $\mathrm{Hf}$ (Figure $5 \mathrm{~b}$ ). The highly fractionated residual magma caused the concentration of these incompatible elements. Therefore, fractional crystallization from the Yanzhiba and Lanbandeng two-mica monzogranites to the barren and $\mathrm{Rb}$-rich pegmatites explains the trace element distribution in the pegmatites in the study area.

\subsection{Tectonic Setting and Petrogenesis of Pegmatite}

As emphasized by Černý, 1991 [2], the trace element signatures of most rare-element pegmatites can be grouped into just two distinctive families: one that is enriched in lithium, cesium, and tantalum (LCT) and the other characterized by enrichment in niobium, yttrium, and fluorine (NYF). In this study, the $\mathrm{Rb}$-rich pegmatites are also variably enriched in $\mathrm{Cs}, \mathrm{Be}, \mathrm{Ta}, \mathrm{Nb}$, and $\mathrm{Sn}$ (Table 1). There are abundant beryl and chrysoberyl (Figure 3c,d). Cassiterite and columbite-group mineral seem to form the most important accessory minerals (Figure 3e,f). Following the classification schemes of Černý (1991) [2] and Černý and Ercit (2005) [4], the Rb-rich pegmatites belong to the LCT family based on the rare element association, and more specifically to the rare element pegmatite class. Based on the rare element mineralogy, they are consistent with the beryl-type (beryl-columbite subtype). As discussed above, the barren and $\mathrm{Rb}$-rich pegmatites are derived by fractionation from the Yanzhiba and Lanbandeng two-mica monzogranites. Therefore, we propose a petrogenetic model of the peraluminous granites and pegmatites, which resulted from decompression melting of the thickened lower crust during a post-collisional extensional tectonic regime. Notably, the post-orogenic setting is also consistent with the required tectonic setting for LCT-type rare-metal pegmatites worldwide [2,4,5].

As noted above, the granitic plutons are extensive in the Ningshan area in the middle segment of SQB (Figure 1b,c). Previous studies have shown that the intrusion of these plutons is considered the result of multi-stage granitic magmatism during the structural evolution of the Qinling Orogenic Belt. 
During this evolution, SQB and SCB experienced a series of subduction, collision, and post-orogenic collapse events along the Mianlue suture [39,47,48,61-64]. Combining the post-collision magmatism and granite-pegmatite genetic relationship, we summarize the petrogenesis of the pegmatites as follows: During extension of the thickened crust, the crust-derived magma moved upward though extensional fractures and along lithological boundaries and was stored in the shallow crust in a magma chamber. The process ultimately gave rise to the formation of peraluminous granites after fractional crystallization and intrusion. The fractionation of these peraluminous granites caused the generation of the affinitive more evolved felsic magma (the residual melts). They continued to intrude into the fissures of early granites and overlying sedimentary rocks and, subsequently, crystallized to form the pegmatites.

\subsection{Importance of Mineral Compositions to Rare Metal Exploration}

Rubidium is highly incompatible in quartz and plagioclase because of its exceptionally large ionic radius and cesium behaves similarly [87]. Quartz, feldspar, and mica are the dominant minerals that crystallize from granitic melts. The extreme fractionation resulting from the extended crystallization of quartz and plagioclase from granitic melts could generate exceptionally high concentrations of $\mathrm{Rb}$ and $\mathrm{Cs}$ in residual melts. The mineral crystallization of the residual melt caused $\mathrm{Rb}$ and $\mathrm{Cs}$ to enter micas and alkali-feldspar because their ionic radius is closer to that of K [87]. In this study, the muscovite and albite are the main $\mathrm{Rb}$-bearing minerals in the $\mathrm{Rb}$-bearing Mus- $\mathrm{Ab}$ pegmatite. The $\mathrm{Rb}$-rich pegmatites are also enriched in $\mathrm{Cs}$, but have low $\mathrm{Li}$ content. In agreement with low $\mathrm{Li}$ content in whole-rock geochemistry, no Li-muscovite and other Li-bearing minerals are observed. This is analogous to that displayed by similar Li-bearing mineral lacking a central intermediate zone of the best-studied LCT-family Tanco pegmatite [91]. As pointed out by Stilling et al. 2006 [91], Li, Cs, and Ta are produced from different zones at Tanco pegmatite. Li and Cs mineralizations may have had different controls [87]. In the case of only barren Mus-Mc-Ab and Rb-rich Mus-Ab pegmatites having been identified, it is difficult to speculate further on the potential distribution of Li mineralization pegmatites in the area.

As emphasized by Selway et al. (2005) [67], the presence of Mn-rich fluorapatite indicates that the pegmatite has economic Li-Cs-Ta potential. The Mn contents of apatite are pointed out to be relatively high in rare metal bearing pegmatites [92,93]. In the spodumene pegmatites from the Tin-Spodumene Belt, with apatite containing up to $3.26 \mathrm{wt} . \% \mathrm{MnO}$ [94], and in the Altay Koktokay no. 3 pegmatites, almost all the apatite contains more than $2.00 \mathrm{wt} . \% \mathrm{MnO}$ [95]. The Mn contents in apatite appear to be related to the $\mathrm{Fe}^{2+} / \mathrm{Fe}^{3+}$ ratios in their melt source and/or the redox conditions during apatite crystallization [92,93]. Miles et al. (2014) [96] presented an equation using Mn content in apatite as an oxybarometer in intermediate and silicic igneous rocks. Their study proposes a negative correlation between $\mathrm{Mn}$ in apatite and oxygen fugacity $\left(\mathrm{fO}_{2}\right)$. However, the relationship of $\mathrm{Mn}$ in apatite as a measure of oxygen fugacity was refuted by Marks et al. (2016), who concluded that the presence of other Mn-bearing phases affecting the Mn content in the apatite [97]. In this study, compared with the $\mathrm{MnO}$ content (0.34-0.54 wt.\%) in apatite from the Mus-Mc-Ab barren pegmatite, the apatite in our study has relatively much higher MnO content (14.51-19.12 wt.\%) from Rb-rich Mus-Ab pegmatite. It can be observed that garnets are abundant in the barren Mus-Mc-Ab pegmatite (Figure $2 \mathrm{~d}, \mathrm{f}$ and Figure 6a). Garnet would incorporate Fe and $\mathrm{Mn}$ into its structure during crystallization. Therefore, the Mn-content in apatite is mostly dependent on the presence garnet or other Fe-Mn minerals. Our results indicate that the Mn-content of apatite may be used to distinguish between barren pegmatites and $\mathrm{Rb}$-rich pegmatites.

Apart from the difference in MnO-content, apatites from $\mathrm{Rb}$-rich Mus-Ab pegmatites have higher $\mathrm{Cl}$ content and lower $\mathrm{F} / \mathrm{Cl}$ ratios in comparison to those of barren Mus-Mc-Ab pegmatites (Figure $8 \mathrm{e}, \mathrm{f}$ ). Previous works demonstrated that halogen contents, especially fluorine and chlorine, could be evaluated using the composition of apatite in igneous rocks $[98,99]$. The reasons for the differences in $\mathrm{Cl}$ contents and $\mathrm{F} / \mathrm{Cl}$ ratios between the $\mathrm{Rb}$-rich Mus- $\mathrm{Ab}$ pegmatites and barren Mus-Mc-Ab pegmatites are 
most likely related to melt compositions. As mentioned above, the Rb-rich pegmatites are crystallized from the more evolved magmas than the barren pegmatites. It should be enriched in $\mathrm{Cl}$ due to the fractionated of the magmas. In summary, the $\mathrm{Mn}$-content and $\mathrm{Cl}$-contents (or $\mathrm{F} / \mathrm{Cl}$ ratios) of apatite could be used as an indicator of the rare metal mineralization potential for the pegmatites in this area.

\section{Conclusions}

(1) The formation age of the barren and Rb-rich pegmatites from Ningshan pegmatite district is 212-203 Ma, which is consistent with the two-mica monzogranites in the middle segment of SQB.

(2) The Rb-rich pegmatite from Ningshan pegmatite district belongs to the LCT family. The magma of the barren and $\mathrm{Rb}$-rich pegmatites originated from the thinned lower crust and formed in a post-collisional environment. The Yanzhiba and Lanbandeng two-mica monzogranites are the potential parental granite of these pegmatites.

(3) The Mn-content in apatite is mostly dependent on the presence garnet or other Fe-Mn minerals. The $\mathrm{Rb}$-rich pegmatite has higher $\mathrm{Mn}$ and $\mathrm{Cl}$ content and lower $\mathrm{F} / \mathrm{Cl}$ ratios than the barren pegmatite, indicating that it can be regarded as an indicator of rare metal mineralization potential.

Author Contributions: Formal analysis, G.W. and Z.L.; project administration, Z.W. and L.C.; writing-original draft, X.N. All authors have read and agreed to the published version of the manuscript.

Funding: This work was financially supported by the National Natural Science Foundation of China, Grant/Award Numbers: 41902042 and 41872092; China Geological Survey, Grant/Award Numbers: 20190437 and 20190613.

Acknowledgments: We are grateful to the editors and anonymous reviewers for their constructive comments, which have considerably improved the manuscript.

Conflicts of Interest: Authors declare no conflict of interest.

\section{References}

1. London, D. Ore-forming processes within granitic pegmatites. Ore Geol. Rev. 2018, 101, 349-383. [CrossRef]

2. Černý, P. Rare-element granitic pegmatites. Part I: Anatomy and internal evolution of pegmatitic deposits. Geosci. Can. 1991, 18, 49-67.

3. Černý, P. Rare-element granitic pegmatites. Part II: Regional to global environments and petrogenesis. Geosci. Can. 1991, 18, 68-81.

4. Černý, P.; Ercit, T.S. The classification of granitic pegmatites revisited. Can. Miner. 2005, 43, 2005-2026.

5. Černý, P.; London, D.; Novák, M. Granitic pegmatites as reflections of their sources. Elements 2012, 8, 289-294. [CrossRef]

6. London, D. Internal differentiation of rare-element pegmatites: A synthesis of recent research. Geol. Soc. Am. Spec. Pap. 1990, 246, 35-50.

7. Jolliff, B.L.; Papike, J.J.; Shearer, C.K. Petrogenetic relationships between pegmatite and granite based on geochemistry of muscovite in pegmatite wall zones, Black Hills, South Dakota, USA. Geochim. Cosmochim. Acta 1992, 56, 1915-1939. [CrossRef]

8. Zhu, Y.F.; Zeng, Y.; Gu, L. Geochemistry of the rare metal-bearing pegmatite No. 3 vein and related granites in the Keketuohai region, Altay Mountains, northwest China. J. Asian Earth Sci. 2006, 27, 61-77. [CrossRef]

9. Webster, J.D.; Thomas, R.; Rhede, D.; Förster, H.-J.; Seltmann, R. Melt inclusions in quartz from an evolved peraluminous pegmatite: Geochemical evidence for strong tin enrichment in fluorine-rich and phosphorus-rich residual liquids. Geochim. Cosmochim. Acta 1997, 61, 2589-2604. [CrossRef]

10. Martin, R.F.; De Vito, C. The patterns of enrichment in felsic pegmatites ultimately depend on tectonic setting. Can. Miner. 2005, 43, 2027-2048. [CrossRef]

11. Lv, Z.H.; Zhang, H.; Tang, Y.; Guan, S.J. Petrogenesis and magmatic-hydrothermal evolution time limitation of Kelumute No. 112 pegmatite in Altay, Northwestern China: Evidence from zircon U-Pb and Hf isotopes. Lithos 2012, 154, 374-391. [CrossRef]

12. Simmons, W.; Falster, A.; Webber, K.; Roda-Robles, E.; Boudreaux, A.P.; Grassi, L.R.; Freeman, G. Bulk Composition of Mt. Mica Pegmatite, Maine, USA: Implications for the Origin of an Lct Type Pegmatite By Anatexis. Can. Miner. 2016, 54, 1053-1070. [CrossRef] 
13. Shaw, R.A.; Goodenough, K.M.; Roberts, N.M.W.; Horstwood, M.S.A.; Chenery, S.R.; Gunn, A.G. Petrogenesis of rare-metal pegmatites in high-grade metamorphic terranes: A case study from the Lewisian Gneiss Complex of north-west Scotland. Precambrian Res. 2016, 281, 338-362. [CrossRef]

14. Zhang, X.; Zhang, H.; Ma, Z.L.; Tang, Y.; Lv, Z.H.; Zhao, J.Y.; Liu, Y.L. A new model for the granite-pegmatite genetic relationships in the Kaluan-Azubai-Qiongkuer pegmatite-related ore fields, the Chinese Altay. J. Asian Earth Sci. 2016, 124, 139-155. [CrossRef]

15. Nabelek, P.I.; Russ-Nabelek, C.; Denison, J. The generation and crystallization conditions of the Proterozoic Harney Peak leucogranite, Black Hills, South Dakota, USA: Petrologic and geochemical constraints. Contrib. Miner. Pet. 1992, 110, 173-191. [CrossRef]

16. Nabelek, P.I.; Russ-Nabelek, C.; Haeussler, G. Stable isotope evidence for the petrogenesis and fluid evolution in the Proterozoic Harney Peak leucogranite, Black Hills, South Dakota. Geochim. Cosmochim. Acta 1992, 56, 403-417. [CrossRef]

17. Robles, E.R.; Perez, A.P.; Roldan, F.V.; Fontan, F. The granitic pegmatites of the Fregeneda area (Salamanca, Spain): Characteristics and petrogenesis. Miner. Mag. 1999, 63, 535-558. [CrossRef]

18. Dill, H. Geology and chemistry of Variscan-type pegmatite systems (SE Germany) —With special reference to structural and chemical pattern recognition of felsic mobile components in the crust. Ore Geol. Rev. 2018, 92, 205-239. [CrossRef]

19. Müller, A.; Romer, R.L.; Pedersen, R.B. The Sveconorwegian pegmatite province-thousands of pegmatites without parental granites. Can. Miner. 2017, 55, 283-315.

20. Webber, K.L.; Simmons, W.B.; Falster, A.U.; Hanson, S.L. Anatectic pegmatites of the Oxford County pegmatite field, Maine, USA. Can. Miner. 2019, 57, 811-815. [CrossRef]

21. Chen, X.J.; Wang, S.R.; Zhang, X.Y. Geological Characteristics of Qinling Granitic Pegmatites; Beijing Geology Publishing House: Beijing, China, 1993; pp. 8-70. (In Chinese)

22. Lu, X.X.; Zhu, C.H.; Gu, D.M.; Zhang, H.M.; Wu, M.; Wu, Y. The main geological and metallogenic characteristics of granitic pegmatite in Eastern Qinling Belt. Geol. Rev. 2010, 56, 21-30. (In Chinese)

23. Feng, Y.; Lei, R.; Ju, M.; Song, G.; Xu, F. Origin and petrogenetic implications of garnet from Rb-rich pegmatites in North Qinling Orogen, China. Geol. J. 2017, 52, 215-237. [CrossRef]

24. Yuan, F.; Liu, J.J.; Carranza, E.J.M.; Zhang, S.; Zhai, D.G.; Liu, G.; Wang, G.W.; Zhang, H.Y.; Sha, Y.Z.; Yang, S.S. Zircon trace element and isotopic $(\mathrm{Sr}, \mathrm{Nd}, \mathrm{Hf}, \mathrm{Pb})$ effects of assimilation-fractional crystallization of pegmatite magma: A case study of the Guangshigou biotite pegmatites from the North Qinling Orogen, central China. Lithos 2018, 302, 20-36. [CrossRef]

25. Yuan, F.; Jiang, S.Y.; Liu, J.; Liu, G.; Zhang, S.; Sha, Y. Origin and evolution of uraniferous pegmatite: A case study from the Xiaohuacha granite-pegmatite system and related country rocks in the Shangdan uranium mineralization district of North Qinling Orogenic Belt, China. Lithos 2020. [CrossRef]

26. Meng, Q.R.; Zhang, G.W. Timing of collision of the North and South China blocks: Controversy and reconciliation. Geology 1999, 27, 123-126. [CrossRef]

27. Meng, Q.R.; Zhang, G.W. Geologic framework and tectonic evolution of the Qinling orogen, central China. Tectonophysics 2000, 323, 183-196. [CrossRef]

28. Yan, Z.; Wang, Z.; Chen, J.; Yan, Q.; Wang, T. Detrital record of Neoproterozoic arc-magmatism along the NW margin of the Yangtze Block, China: U-Pb geochronology and petrography of sandstones. J. Asian Earth Sci. 2010, 37, 322-334. [CrossRef]

29. Lai, S.; Qin, J.; Chen, L.; Grapes, R. Geochemistry of ophiolites from the Mian-Lue Suture Zone: Implications for the tectonic evolution of the Qinling Orogen, Central China. Int. Geol. Rev. 2008, 50, 650-664. [CrossRef]

30. Dong, Y.; Zhang, G.; Neubauer, F.; Liu, X.; Genser, J.; Hauzenberger, C. Tectonic evolution of the Qinling orogen, China: Review and synthesis. J. Asian Earth Sci. 2011, 41, 213-237. [CrossRef]

31. Zhang, G.; Meng, Q.; Lai, S. Tectonics and structure of Qinling orogenic belt. Sci. China (Sci. Sin.) Ser. B. 1995, 11, 1379-1394.

32. Zhang, G.; Meng, Q.; Yu, Z.; Sun, Y.; Zhou, D.; Guo, A. Orogenesis and dynamics of the Qinling orogen. Sci. China Ser. D-Earth Sci. 1996, 39, 225-234.

33. Zhang, G.; Dong, Y.; Lai, S.; Guo, A.; Meng, Q.; Liu, S.; Cheng, S.; Yao, A.; Zhang, Z.; Pei, X. Mianlue tectonic zone and Mianlue suture zone on southern margin of Qinling-Dabie orogenic belt. Sci. China Ser. D-Earth Sci. 2004, 47, 300-316. [CrossRef] 
34. Mattauer, M.; Matte, P.; Malavieille, J.; Tapponnier, P.; Maluski, H.; Qin, X.Z.; Lun, L.Y.; Qin, T.Y. Tectonics of the Qinling belt: Build-up and evolution of eastern Asia. Nature 1985, 317, 496-500. [CrossRef]

35. Zhang, G.W.; Zhang, Z.Q.; Dong, Y.P. Nature of main tectono-lithostratigraphic units of the Qinling Orogen: Implications for the tectonic evolution. Acta Pet. Sin. 1995, 11, 101-114.

36. Liu, S.W.; Li, Q.G. Petrogenesis of Indosinian Granitoids in Middle-Segment of South Qinling Tectonic Belt:Constraints from Sr-Nd Isotopic Systematics. Acta Geol. Sin. Engl. Ed. 2011, 85, 610-628. [CrossRef]

37. Wang, X.; Tao, W.; Zhang, C. Neoproterozoic, Paleozoic, and Mesozoic granitoid magmatism in the Qinling Orogen, China: Constraints on orogenic process. J. Asian Earth Sci. 2013, 72, 129-151. [CrossRef]

38. Li, N.; Chen, Y.J.; Santosh, M.; Pirajno, F. Compositional polarity of Triassic granitoids in the Qinling Orogen, China: Implication for termination of the northernmost paleo-Tethys. Gondwana Res. 2015, 27, $244-257$. [CrossRef]

39. Dong, Y.; Liu, X.; Zhang, G.; Chen, Q.; Zhang, X.; Wei, L.; Chen, Y. Triassic diorites and granitoids in the Foping area: Constraints on the conversion from subduction to collision in the Qinling orogen, China. J. Asian Earth Sci. 2012, 47, 123-142. [CrossRef]

40. Zhang, H.F.; Ouyang, J.P.; Ling, W.L.; Chen, Y.L. Pb, Sr, Nd isotope composition of Ningshan Granitoids, South Qiling and their deep geological information. Acta Pet. Miner. 1997, 16, 22-32. (In Chinese)

41. Peng, H.L.; Li, W.C.; Li, W.J.; Wang, J.J.; Lv, J.L.; Du, B. Geological characteristics and prospecting potential of Rubidium and other rare metal mineralization in Ninghan county, Shanxi province. Geol. Shanxi. 2016, 34, 21-26. (In Chinese)

42. Liu, Y.; Hu, Z.; Gao, S.; Günther, D.; Xu, J.; Gao, C.; Chen, H. In situ analysis of major and trace elements of anhydrous minerals by LA-ICP-MS without applying an internal standard. Chem. Geol. 2008, 257, $34-43$. [CrossRef]

43. Liu, Y.; Hu, Z.; Zong, K.; Gao, C.; Gao, S.; Xu, J.; Chen, H. Reappraisement and refinement of zircon U-Pb isotope and trace element analyses by LA-ICP-MS. Chin. Sci. Bull. 2010, 55, 1535-1546. [CrossRef]

44. Hou, K.; Li, Y.; Tian, Y. In situ U-Pb zircon dating using laser ablation-multi ion counting-ICP-MS. Miner. Depos. 2009, 28, 481-492.

45. Maniar, P.D.; Piccoli, P.M. Tectonic discrimination of granitoids. Geol. Soc. Am. Bull. 1989, 101, 635-643. [CrossRef]

46. Sun, S.S.; Mcdonough, W.F. Chemical and isotopic systematics of oceanic basalts: Implications for mantle composition and processes. Geol. Soc. Lond. Spec. Pub. 1989, 42,313-345. [CrossRef]

47. Jiang, Y.H.; Jin, G.D.; Liao, S.Y.; Zhou, Q.; Zhao, P. Geochemical and Sr-Nd-Hf isotopic constraints on the origin of Late Triassic granitoids from the Qinling orogen, central China: Implications for a continental arc to continent-continent collision. Lithos 2010, 117, 183-197. [CrossRef]

48. Yang, P.; Liu, S.; Li, Q.; Wang, Z.; Wang, R.; Wang, W. Geochemistry and zircon U-Pb-Hf isotopic systematics of the Ningshan granitoid batholith, middle segment of the south Qinling belt, Central China: Constraints on petrogenesis and geodynamic processes. J. Asian Earth Sci. 2012, 61, 166-186. [CrossRef]

49. Tischendorf, G.; Gottesmann, B.; Foerster, H.J.; Trumbull, R.B. On Li-bearing micas: Estimating Li from electron microprobe analyses and an improved diagram for graphical representation. Miner. Mag. 1997, 61, 809-834. [CrossRef]

50. Van Lichtervelde, M.; Grégoire, M.; Linnen, R.L.; Béziat, D.; Salvi, S. Trace element geochemistry by laser ablation ICP-MS of micas associated with Ta mineralization in the Tanco pegmatite, Manitoba, Canada. Contrib. Miner. Pet. 2008, 155, 791-806. [CrossRef]

51. Tindle, A.G.; Webb, P.C. Estimation of lithium contents in trioctahedral micas using microprobe data: Application to micas from granitic rocks. Eur. J. Miner. 1990, 2, 595-610. [CrossRef]

52. Mezger, K.; Krogstad, E. Interpretation of discordant U-Pb zircon ages: An evaluation. J. Metamorph. Geol. 1997, 15, 127-140. [CrossRef]

53. Geisler, T.; Schaltegger, U.; Tomaschek, F. Re-equilibration of zircon in aqueous fluids and melts. Elements 2007, 3, 43-50. [CrossRef]

54. Soman, A.; Geisler, T.; Tomaschek, F.; Grange, M.; Berndt, J. Alteration of crystalline zircon solid solutions: A case study on zircon from an alkaline pegmatite from Zomba-Malosa, Malawi. Contrib. Miner. Pet. 2010, 160, 909-930. [CrossRef] 
55. Zhou, Q.; Qin, K.; Tang, D.; Wang, C.; Sakyi, P.A. LA-ICP-MS U-Pb zircon, columbite-tantalite and ${ }^{40} \mathrm{Ar}-{ }^{39} \mathrm{Ar}$ muscovite age constraints for the rare-element pegmatite dykes in the Altai orogenic belt, NW China. Geol. Mag. 2018, 155, 707-728. [CrossRef]

56. Zhou, Q.; Qin, K.; Tang, D.; Tian, Y.; Cao, M.; Wang, C. Formation Age and Evolution Time Span of the Koktokay No. 3 Pegmatite, Altai, NW C hina: Evidence from U-Pb Zircon and ${ }^{40} \mathrm{Ar}-{ }^{39} \mathrm{Ar}$ Muscovite Ages. Resour. Geol. 2015, 65, 210-231. [CrossRef]

57. Wang, C.L.; Qin, K.Z.; Tang, D.M.; Zhou, Q.F.; Shen, D.M.; Guo, Z.L.; Guo, X.J. Geochronology and Hf isotope of zircon for the Arskartor Be-Nb-Mo deposit in Altay and its geological implications. Acta Pet. Sin. 2015, 2337-2352. (In Chinese)

58. Hoskin, P.W.; Schaltegger, U. The composition of zircon and igneous and metamorphic petrogenesis. Rev. Miner. Geochem. 2003, 53, 27-62. [CrossRef]

59. Wang, T.; Tong, Y.; Jahn, B.M.; Zou, T.R.; Wang, Y.B.; Hong, D.W.; Han, B.F. SHRIMP U-Pb Zircon geochronology of the Altai No. 3 Pegmatite, NW China, and its implications for the origin and tectonic setting of the pegmatite. Ore Geol. Rev. 2007, 32, 325-336. [CrossRef]

60. Yang, H.; Wang, W.; Liu, J.H. Zircon U-Pb dating and its geological significance of granitic pegmatites from the Kuandian and Sanjiazi area in Eastern Liaoning Province. Acta Pet. Sin. 2017, 9, 2675-2688. (In Chinese)

61. Qin, J.F.; Lai, S.C.; Wang, J.; Li, Y.F. High-Mg\# adakitic tonalite from the Xichahe area, South Qinling orogenic belt (central China): Petrogenesis and geological implications. Int. Geol. Rev. 2007, 49, 1145-1158.

62. Qin, J.; Lai, S.; Wang, J.; Li, Y. Zircon LA-ICP MS U-Pb age, Sr-Nd-Pb isotopic compositions and geochemistry of the Triassic post-collisional Wulong adakitic granodiorite in the South Qinling, Central China, and its petrogenesis. Acta Geol. Sin. Engl. Ed. 2008, 82, 425-437.

63. Yang, P.; Liu, S.; Li, Q.; Fan, Z.; Wang, Z.; Wang, D.; Wang, R.; Yan, Q.; Zhen, Y. Ages of the Laocheng Granitoids and Crustal Growth in the South Qinling Tectonic Domain, Central China: Zircon U-Pb and Lu-Hf Isotopic Constraints. Acta Geol. Sin. Engl. Ed. 2011, 85, 854-869. [CrossRef]

64. Qin, J.F.; Lai, S.C.; Li, Y.F. Multi-stage granitic magmatism during exhumation of subducted continental lithosphere: Evidence from the Wulong pluton, South Qinling. Gondwana Res. 2013, 24, 1108-1126. [CrossRef]

65. Zhang, F.; Liu, S.; Xu, C.; Li, Q.; Dai, J.; Yang, K.; Wu, F.; Chen, Y. Xiba granitic pluton in the Qinling orogenic belt, central China: Its petrogenesis and tectonic implications. Acta Geol. Sin. Engl. Ed. 2012, 86, 1128-1142.

66. Dill, H.G. Pegmatites and aplites: Their genetic and applied ore geology. Ore Geol. Rev. 2015, 69, 417-561. [CrossRef]

67. Selway, J.B.; Breaks, F.W.; Tindle, A.G. A review of rare-element (Li-Cs-Ta) pegmatite exploration techniques for the Superior Province, Canada, and large worldwide tantalum deposits. Explor. Min. Geol. 2005, 14, 1-30. [CrossRef]

68. Halliday, A.; Davidson, J.P.; Hildreth, W.; Holden, P. Modelling the petrogenesis of high Rb/Sr silicic magmas. Chem. Geol. 1991, 92, 107-114. [CrossRef]

69. Gamage, S.J.K.; Rupasinghe, M.S.; Dissanayake, C.B. Application of Rb-Sr ratios to gem exploration in the granulite belt of Sri Lanka. J. Geochem. Explor. 1992, 43, 281-292. [CrossRef]

70. Raimbault, L.; Cuney, M.; Azencott, C.; Duthou, J.L.; Joron, J.L. Geochemical evidence for a multistage magmatic genesis of Ta-Sn-Li mineralization in the granite at Beauvoir, French Massif Central. Econ. Geol. 1995, 90, 548-576. [CrossRef]

71. Xie, L.; Wang, R.C.; Zhu, J.C.; Lu, J.J.; Zhang, W.L.; Che, X.D.; Zhang, R.Q.; Huang, F.F. Felsic dykes in the metallogenic area of southern Hunan Province and their implications for mineralization and exploration. Acta Pet. Sin. 2013, 29, 4261-4280. (In Chinese)

72. Linnen, R.L.; Keppler, H. Melt composition control of $\mathrm{Zr} / \mathrm{Hf}$ fractionation in magmatic processes. Geochim. Cosmochim. Acta 2002, 66, 3293-3301. [CrossRef]

73. Li, J.; Huang, X.L.; He, P.L.; Li, W.X.; Yu, Y.; Chen, L.L. In situ analyses of micas in the Yashan granite, South China: Constraints on magmatic and hydrothermal evolutions of $\mathrm{W}$ and $\mathrm{Ta}-\mathrm{Nb}$ bearing granites. Ore Geol. Rev. 2015, 65, 793-810. [CrossRef]

74. Bau, M. Controls on the fractionation of isovalent trace elements in magmatic and aqueous systems: Evidence from $\mathrm{Y} / \mathrm{Ho}, \mathrm{Zr} / \mathrm{Hf}$, and lanthanide tetrad effect. Contrib. Miner. Pet. 1996, 123, 323-333. [CrossRef]

75. Nash, W.P.; Crecraft, H.R. Partition coefficients for trace elements in silicic magmas. Geochim. Cosmochim. Acta 1985, 49, 2309-2322. [CrossRef] 
76. Rollinson, H. Using Geochemical Data: Evaluation, Presentation, Interpretation; Longman Geochemistry Society: London, UK, 1993; pp. 1-352.

77. Bea, F. Residence of REE, Y, Th and U in granites and crustal protoliths; implications for the chemistry of crustal melts. J. Pet. 1996, 37, 521-552. [CrossRef]

78. Shao, T.Q.; Jiang, K.T.; Liu, Y.H.; Tang, H.H.; Qin, J.C.; Hu, B.; Wang, Q.; Zhang, Y.N.; Zhang, H. Geochemistry and a metallogenic model for $\mathrm{Nb}$-Ta-bearing granitic pegmatites from the northern Qaidam Basin. Geol. J. 2018, 53, 113-123. [CrossRef]

79. Fei, G.C.; Menuge, J.F.; Li, Y.Q.; Yang, J.Y.; Deng, Y.; Chen, C.S.; Yang, Y.F.; Yang, Z.; Qin, L.Y.; Zheng, L. Petrogenesis of the Lijiagou spodumene pegmatites in Songpan-Garze Fold Belt, West Sichuan, China: Evidence from geochemistry, zircon, cassiterite and coltan UPb geochronology and Hf isotopic compositions. Lithos 2020, 364-365. [CrossRef]

80. Möller, P.; Muecke, G.K. Significance of Europium anomalies in silicate melts and crystal-melt equilibria: A re-evaluation. Contrib. Miner. Pet. 1984, 87, 242-250. [CrossRef]

81. D'Arco, P.; Piriou, B. Fluorescence spectra of $\mathrm{Eu}^{3+}$ in synthetic polycrystalline anorthite: Distribution of Eu $\mathrm{Eu}^{3+}$ in the structure. Am. Miner. 1989, 74, 191-199.

82. Kimata, M. The crystal structure of non-stoichiometric Eu-anorthite: An explanation of the Eu-positive anomaly. Miner. Mag. 1988, 52, 257-265. [CrossRef]

83. Walker, R.J.; Hanson, G.N.; Papike, J.J.; O'neil, J.R.; Laul, J.C. Internal evolution of the Tin Mountain pegmatite, Black Hills, South Dakota. Am. Miner. 1986, 71, 440-459.

84. Michallik, R.M.; Wagner, T.; Fusswinkel, T.; Heinonen, J.S.; Heikkilä, P. Chemical evolution and origin of the Luumäki gem beryl pegmatite: Constraints from mineral trace element chemistry and fractionation modeling. Lithos 2017, 274, 147-168. [CrossRef]

85. Černý, P.; Masau, M.; Goad, B.E.; Ferreira, K. The Greer Lake leucogranite, Manitoba, and the origin of lepidolite-subtype granitic pegmatites. Lithos 2005, 80, 305-321. [CrossRef]

86. Roda, E.; Pesquera, A.; Fontan, F.; Keller, P. Phosphate mineral associations in the Canada pegmatite (Salamanca, Spain): Paragenetic relationships, chemical compositions, and implications for pegmatite evolution. Am. Miner. 2004, 89, 110-125. [CrossRef]

87. Linnen, R.L.; Van Lichtervelde, M.; Černý, P. Granitic pegmatites as sources of strategic metals. Elements 2012, 8, 275-280. [CrossRef]

88. Bea, F.; Fershtater, G.; Corretgé, L. The geochemistry of phosphorus in granite rocks and the effect of aluminium. Lithos 1992, 29, 43-56. [CrossRef]

89. Blundy, J.D.; Wood, B.J. Crystal-chemical controls on the partitioning of Sr and Ba between plagioclase feldspar, silicate melts, and hydrothermal solutions. Geochim. Cosmochim. Acta 1991, 55, 193-209. [CrossRef]

90. Chappell, B. Aluminium saturation in I-and S-type granites and the characterization of fractionated haplogranites. Lithos 1999, 46, 535-551. [CrossRef]

91. Stilling, A.; Černý, P.; Vanstone, P.J. The Tanco pegmatite at Bernic Lake, Manitoba. XVI. Zonal and bulk compositions and their petrogenetic significance. Can. Miner. 2006, 44, 599-623. [CrossRef]

92. Sha, L.K.; Chappell, B.W. Apatite chemical composition, determined by electron microprobe and laser-ablation inductively coupled plasma mass spectrometry, as a probe into granite petrogenesis. Geochim. Cosmochim. Acta 1999, 63, 3861-3881. [CrossRef]

93. Belousova, E.; Griffin, W.; O’Reilly, S.Y.; Fisher, N. Apatite as an indicator mineral for mineral exploration: Trace-element compositions and their relationship to host rock type. J. Geochem. Explor. 2002, 76, 45-69. [CrossRef]

94. Swanson, S.E. Mineralogy of spodumene pegmatites and related rocks in the tin-spodumene belt of North Carolina and South Carolina, USA. Can. Miner. 2012, 50, 1589-1608. [CrossRef]

95. Cao, M.J.; Zhou, Q.F.; Qin, K.Z.; Tang, D.M.; Evans, N.J. The tetrad effect and geochemistry of apatite from the Altay Koktokay No. 3 pegmatite, Xinjiang, China: Implications for pegmatite petrogenesis. Miner. Pet. 2013, 107, 985-1005. [CrossRef]

96. Miles, A.; Graham, C.; Hawkesworth, C.; Gillespie, M.; Hinton, R.; Bromiley, G. Apatite: A new redox proxy for silicic magmas? Geochim. Cosmochim. Acta 2014, 132, 101-119. [CrossRef]

97. Marks, M.A.; Scharrer, M.; Ladenburger, S.; Markl, G. Comment on" Apatite: A new redox proxy for silicic magmas?"[Geochimica et Cosmochimica Acta 132 (2014) 101-119]. Geochim. Cosmochim. Acta 2016, 183, 267-270. [CrossRef] 
98. Mathez, E.A.; Webster, J.D. Partitioning behavior of chlorine and fluorine in the system apatite-silicate melt-fluid. Geochim. Cosmochim. Acta 2005, 49, 1275-1286. [CrossRef]

99. Webster, J.D.; Tappen, C.M.; Mandeville, C.M. Partitioning behavior of chlorine and fluorine in the system apatite-melt-fluid. II: Felsic silicate systems at 200 MPa. Geochim. Cosmochim. Acta 2009, 73, 559-581. [CrossRef]

(C) 2020 by the authors. Licensee MDPI, Basel, Switzerland. This article is an open access article distributed under the terms and conditions of the Creative Commons Attribution (CC BY) license (http://creativecommons.org/licenses/by/4.0/). 